\title{
Über den intermediären Kohlehydratstoffwechsel beim experimentellen Fieber.
}

\author{
Von \\ Kesae Adachi und Shintaro Kasai. \\ (姿達今朝街) (笠井慥太郎) \\ (Aus der Medizinischen Klinik von Prof. Di: T. Kato, \\ Kaiserliche Tohoku Universität zu Sendai.)
}

Einer von uns (A dachi) ${ }^{1)}$ hat am gesunden Hunde den Milchsäurestoff wechsel im Muskel beim durch Naphthylamininjektion hervorgerufenen Fieber untersucht und daraus gefolgert, dass die Vermehrung der Blutmilchsäure auf Mobilisierung des Leberglykogens zurückgeführt werden dürfte. Wir haben diesmal den vorliegenden Versuch gemacht, um die Veränderungen des intermediären Kohlehydratstoffwechsels beim Naphthylaminfieber, das auf chemischem Wege durch sympathischen Reiz sekundär erzeugt wird, und die beim Wärmestichfieber, das durch direkte mechanische Einwirkung auf das Wärmezentrum bewirkt wird, miteinander zu vergleichen.

Für den Versuch wurden immer wohlgenährte gesunde Kaninchen benutzt, die 2-3 Wochen lang mit einem bestimmten Futter (Tofukara und Mohrrübe) ernährt wurden und 12 Stunden vor dem Versuch hungern mussten; früh am Morgen fesselte man die Tiere auf dem Tierhalter, das vorher elektrisch auf $38^{\circ}-39^{\circ} \mathrm{C}$ erwärmt war, bedeckte sie sorgfältig, damit sie keine Wärme einbüssten, und legte an einem Hinterbein die Femoralarterie bloss, in deren zentrales Ende eine Kanüle eingeschoben wurde, um jederzeit Blut entnehmen zu können.

Die Körpertemperatur wurde im Rektum gemessen, der Blutzuckergehalt nach der $\mathrm{H}$ aged orn $\mathrm{u}$. Jen sen schen ${ }^{2)}$ Methode und der Blutmilchsäuregehalt nach der von $\mathrm{H}$ a yas a $\mathrm{k} a \mathrm{u}$. In a wa sh ir $\mathrm{o}^{32}$ aus unserer Klinik modifizierten Anrep u. Can 1 an schen ${ }^{4}$ Methode quantitativ bestimmt. Die jedesmal entnommene Blutmenge betrug $1,5 \mathrm{ccm}$,

1) A d a chi, Tohoku Journ. Exp. Med., 1932, 20, 93.

2) Hagedorn u. Jensen, Bioch. Reitschr, 1923, 135, 46.

3) Hay a s ka u. In aw ashiro, Tohoku Journ. Exp. Med., 1928, 12, 1.

4) Anrepu. Cannan, Journ. Physiol., 1923, 58, 244. 
wovon, $1 \mathrm{ccm}$ zur quantitativen Bestimmung der Blutmilchsäure und die übrige zur Blutzuckerbestimmung gebraucht wurde.

\section{I. Über den Einfluss der Fesselung auf den inter- mediären Kohlehydratstoffwechsel.}

Weil bei diesem Fieberversuch das Versuchstier, wie oben erwähnt, auf dem Tierhalter gefesselt und so während verhältnismässig langer Zeit in unnatürlichem Zustand beobachtet wird, so musste ein Kontrollversuch gemacht werden, um vor allem festzustellen, wie die Fesselung die Körpertemperatur und den Blutzucker- und Blutmilchsäuregehalt beeinflusst.

Was die Ordnung der Versuchsbehandlung betrifft, so zeichneten wir zuerst die Körpertemperatur des Kaninchens unmittelbar vor dem Fesseln auf, gleich darauf fesselten wir es, oben angegeben, entnahm ihm zum ersten Mal Blut, sobald die Vorbereitung dafür fertig war, und entnahm dann ebenso stündlich Blut, und zwar 5 Stunden lang, wobei wir am betreffenden Blut Blutzucker und -milchsäure quantitativ bestimmten und die Körpertemperatur bei jeder Blutentnahme massen; bei Versuch $6 \mathrm{u}$. 7, deren Nervus auricularis einer Ohrmuschel und deren Halssympathicus auf derselben Seite einige Tage vor dem Versuch durchschnitten waren, entnahmen wir das Blut unmittelbar vor dem Fesseln aus der mitten in der Ohrmuschel verlaufenden Arterie zur quantitativen Bestimmung des Blutzucker- und Blutmilchsäuregehalts.

Obwohl das Tier, wie oben erwähnt, auf einem Tierhalter, der vorher in einer seiner Körpertemperatur angenäherten Wärme, d.h. bei $38^{\circ}-39^{\circ} \mathrm{C}$ gehalten war, gefesselt lag, so doch sank seine Körpertemperatur nach dem Fesseln allmählich und erreichte nach $1 \frac{1}{2}-3$ Stunden den niedrigsten Wert. Danach stieg sie wieder allmählich, aber erreichte meist 5 Stunden nach dem Fesseln noch nicht wieder seinen Anfangswert.

B o $\mathrm{k}^{5}$ ) meinte, dass die Körpertemperatur des Kaninchens durch die Aussentemperatur erheblich beeinflusst wird, B o e hm $\mathrm{u}$. $\mathrm{H}$ off $\mathrm{mann},{ }^{\left({ }^{(6)}\right.} \mathrm{Fu} \mathrm{jii}{ }^{i)}$ und Voin a $^{(8)}$ beobachteten, dass die Körpertemperatur der Katze und des Kaninchens in Zimmertemperatur beim Fesseln sinkt, und Lic h t9) glaubte, dass die Ursache der Körpertemperatursenkung in der Abuahme der Wärmebildung liege.

5) Bock, Arch. f. exp. Pathol. u. Pharmakol., 1912, 68, 1.

6) Boehm u. Hoff mann, Arch. f. exp. Pathol. u. Pharmakol., 1878, 8, 375.

7) F u jii, Tohoku Journ. Exp. Med., 1921, 2, 9.

8) Vo i n ar, Zurnal eksperimemental'noj biologü i medicing, 1928, 9, 409; ref. in Berichte über die ges. Physiol., 1928, 46, 667.

9) Lich t, Med. Kl., 1927, 1923. 
Tabelle 1.

Über den Einfluss der Fesselung auf die Blutmilchsäure u. den Blutzucker.

\begin{tabular}{|c|c|c|c|c|c|c|c|}
\hline \multirow[b]{2}{*}{ Nr. } & \multirow{2}{*}{$\begin{array}{c}\text { Körper- } \\
\text { gew. u. } \\
\text { Geschlecht }\end{array}$} & \multirow{2}{*}{$\begin{array}{l}\text { Vor u. nach der } \\
\text { Fesselung }\end{array}$} & \multirow{2}{*}{$\begin{array}{c}\text { Körper- } \\
\text { temp. } \\
\mathrm{C}^{\mathrm{o}}\end{array}$} & \multicolumn{2}{|c|}{ Blutnilchsäure } & \multicolumn{2}{|c|}{ Blutzucker } \\
\hline & & & & $\mathrm{mg} / \mathrm{dl}$ & $\begin{array}{c}\text { Diff. in } \\
\%\end{array}$ & $\mathrm{mg} / \mathrm{dl}$ & $\begin{array}{l}\text { Diff. in } \\
\%\end{array}$ \\
\hline 1 & $\begin{array}{c}2372 \mathrm{~g} \\
\text { 우 }\end{array}$ & $\begin{array}{l}\text { Vor } \\
1 \text { 30' nach } \\
1 \text { St. 30' " } \\
2 \text { St. 30' " } \\
\text { 3 St. 30' " } \\
4 \text { St. 30' ", } \\
5 \text { St. 30' ", }\end{array}$ & $\begin{array}{l}38,6 \\
38,0 \\
37,2 \\
37,3 \\
37,8 \\
37,9 \\
37,7\end{array}$ & $\begin{array}{l}33,43 \\
16,71 \\
14,14 \\
16,71 \\
20,57 \\
18,00\end{array}$ & & $\begin{array}{l}146 \\
172 \\
163 \\
182 \\
170 \\
181\end{array}$ & \\
\hline 2 & $\begin{array}{c}1852 g \\
\text { 의 }\end{array}$ &  & $\begin{array}{l}39,2 \\
39,2 \\
38,3 \\
38,0 \\
38,0 \\
38,2 \\
38,6\end{array}$ & $\begin{array}{l}51,43 \\
25,71 \\
18,00 \\
18,00 \\
16,71 \\
18,00\end{array}$ & & $\begin{array}{l}102 \\
161 \\
172 \\
141 \\
132 \\
124\end{array}$ & \\
\hline $\mathbf{3}$ & $\begin{array}{c}2108 \mathrm{~g} \\
\text { 우 }\end{array}$ & $\begin{array}{l}\text { Vor } \\
\quad 20^{\prime} \text { naeh } \\
1 \text { St. } 20^{\prime} \quad ", \\
2 \text { St. } 20^{\prime} \quad ", \\
3 \text { St. } 20^{\prime} \quad ", \\
4 \text { St. } 20^{\prime} \quad ", \\
5 \text { St. } 20^{\prime} \quad ",\end{array}$ & $\begin{array}{l}39,0 \\
39,0 \\
38,1 \\
38,0 \\
38,4 \\
38,8 \\
38,7\end{array}$ & $\begin{array}{l}79,71 \\
37,29 \\
21,86 \\
18,00 \\
18,00 \\
19,29\end{array}$ & & $\begin{array}{l}111 \\
138 \\
139 \\
127 \\
132 \\
134\end{array}$ & \\
\hline 4 & $\begin{array}{c}1800 \mathrm{~g} \\
\delta\end{array}$ & 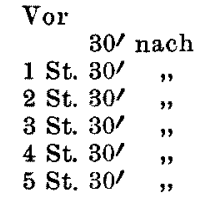 & $\begin{array}{l}38,9 \\
38,7 \\
38,0 \\
38,0 \\
38,3 \\
38,5 \\
38,2\end{array}$ & $\begin{array}{l}32,14 \\
18,00 \\
18,00 \\
18,00 \\
17,35 \\
18,00\end{array}$ & & $\begin{array}{l}106 \\
113 \\
119 \\
143 \\
145 \\
163\end{array}$ & \\
\hline 5 & $\begin{array}{l}2180 \mathrm{~g} \\
\quad \text { 우 } \\
\end{array}$ & $\begin{array}{l}\text { Vor } \\
10^{\prime} \text { nach } \\
1 \text { St. } 10^{\prime} ", \\
2 \text { St. } 10^{\prime} ", \\
\text { 3 St. } 10^{\prime} ", \\
4 \text { St. } 10^{\prime} ", \\
5 \text { St. } 10^{\prime} \quad "\end{array}$ & $\begin{array}{l}39,4 \\
38,7 \\
38,1 \\
37,2 \\
37,0 \\
37,0 \\
38,0\end{array}$ & $\begin{array}{l}56,58 \\
34,71 \\
16,71 \\
21,57 \\
23,14 \\
23,14\end{array}$ & & $\begin{array}{l}148 \\
136 \\
124 \\
177 \\
186 \\
168\end{array}$ & \\
\hline 6 & $1770 \mathrm{~g}$ & $\begin{array}{l}\text { Vor } \\
10^{\prime} \text { nach } \\
1 \text { St. } 10^{\prime} ", \\
2 \text { St. } 10^{\prime} \quad " \\
3 \text { St. 10' ", } \\
\text { 4 St. 10' ", }\end{array}$ & $\begin{array}{l}38,3 \\
38,3 \\
37,2 \\
36,7 \\
36,6 \\
36,8\end{array}$ & $\begin{array}{l}14,14 \\
23,14 \\
16,71 \\
13,51 \\
14,14 \\
13,51\end{array}$ & $\begin{array}{l}+64 \\
+18 \\
-\quad 4 \\
\pm \quad 0 \\
-\quad 4\end{array}$ & $\begin{array}{l}132 \\
141 \\
146 \\
170 \\
195 \\
195\end{array}$ & $\begin{array}{l}+7 \\
+11 \\
+29 \\
+48 \\
+48\end{array}$ \\
\hline 7 & $\begin{array}{c}1810 \mathrm{~g} \\
\text { 우 }\end{array}$ & 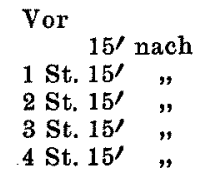 & $\begin{array}{l}39,2 \\
38,9 \\
38,3 \\
38,5 \\
38,5 \\
38,4\end{array}$ & $\begin{array}{l}21,86 \\
39,86 \\
25,08 \\
21,86 \\
19,96 \\
21,86\end{array}$ & $\begin{array}{r}+82 \\
+15 \\
\pm 0 \\
\pm \quad 9 \\
\pm 0\end{array}$ & $\begin{array}{l}127 \\
136 \\
141 \\
143 \\
177 \\
190\end{array}$ & $\begin{array}{l}+7 \\
+11 \\
+13 \\
+39 \\
+50\end{array}$ \\
\hline
\end{tabular}




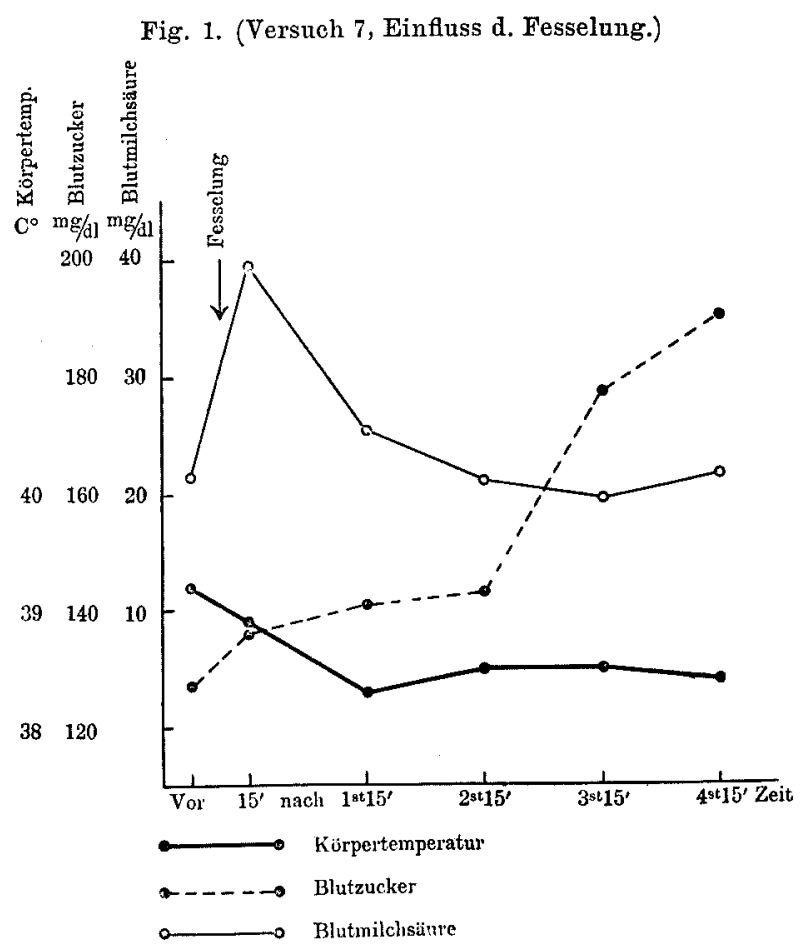

Seitdem Boehm u. Hoffmann beträchtliche $\mathrm{Hy}$ perglykämie und Glykosurie nach Fesseln beobachtet hatten, stellten sie auch Freund u. Marchand, ${ }^{10}$ Jacobsen, ${ }^{11}$ Hirsch u. Reinbach, $\left.{ }^{12}\right)$ Stenström ${ }^{13)}$ und Fujiil fest. Jacobsen führte die Fesselungshyperglykämie auf den Schreck zurück. Nach Fuji i s ${ }^{i}$ Untersuchung steht Fesselungshyperglykämie in inniger $\mathrm{Be}$ ziehung zur Senkung der Körpertemperatur, diese aber verläuft nicht immer parallel zur

Zunahme des Blutzuckers; er beobachtete, dass beim Kaninchen mit erzeugter Fesselungshyperglykämie die chromaphile Substanz der Nebeuniere abnahm und beim splanchnikotomierten, Fesselungshyperglykämie in sehr geringem Grade eintrat. Hiray a m $\mathrm{a}^{14}$ ) berichtet, dass beim Kaninchen mit beiderseitig entfernten Nebenneieren der Grad der eintretenden Fesselungshyperglykämie sehr leicht ist.

Auch bei unserem Versuch vermehrte sich der Blutzuckerwert schon 10 Minuten nach dem Fesseln und erreichte seinen Höhepunkt frühestens $2 \frac{1}{4}$ Stunden, meistens aber 4-5 Stunden nach dem Fesseln, was mit den Ergebnissen obiger Autoren übereinstimmt.

Kürzlich beobachtete Kawa mura, ${ }^{15}$ ) dass Blutmilchsäure nach dem Fesseln beträchtlich zunahm und die vermehrte Blutmilchsäure 3 Stunden nach dem Fesseln auf den Wert in Ruhe zurückkam, danach aber allmählich wieder zunahm, und dass beim splanchnikotomierten Kaninchen keine Fesselungshyperlactazidämie eintrat.

10) Freund u. Ma rehand, Arch. f. exp. Pathol. u. Pharmakol., 1912, 72, 56.

11) J a cobse n, Bioch. Zeitschr., 1913, 51, 443.

12) Hirsch u. Reinbach, Hoppe-Seyler's Zeitschr., 1913, 87, 122.

13) Stenström, Bioch. Zeitschr., 1914, 58, 472.

14) Hir a y a ma, Tohoku Journ. Exp. Med., 1926-27, 8, 37

15) Kaw a mura, Kyoto Ikadaigaku Zasshi, 1928, 2, 161. 
Bei unserem Versuch stieg der Milchsäuregehalt des Bluts nach der Fesselung und der Blutentnahme beträchtlich, z. B. betrug er bei Versuch 3 ungefähr $80 \mathrm{mg} / \mathrm{dl}$, nahm bei Versuch $6 \mathrm{u}$. 7, bei denen er vor dem Fesseln nur ca. $20 \mathrm{mg} / \mathrm{dl}$ war, nach dem Fesseln betrïchtlich zu und kam 2 Stunden danach auf den Wert in Ruhe zurück. Später schwankte er nur noch sehr wenig.

Diese Zunahme des Blutzucker- und Blutmilchsäuregehalts infolge des Fesselns muss ihre Ursache darin haben, dass das Tier, wie $\mathrm{Fujii}^{7)}$ und Kaw a mura ${ }^{15)}$ beobachteten, beim Fesseln sehr aufgeregt wird, wobei die zentrale Nervenreizung über den Splanchnicus einerseits auf die Leber und andererseits auf die Nebenniere einwirkt und Hyperlaktazidämie und Adrenalinhyperglykämie verursacht. Dass Adrenalin Zunahme der Blutmilchsäure hervorruft, haben schon Feinchemid u. Ferdmann ${ }^{16)}$ Hayasaka $^{17)}$ u. a. festgestellt. Wenn diese beim Fesseln so vermehrte Blutmilchsäure dabei aus der Muskelbewegung entstehen könnte, so müsste sie auf Grund vieler Versuchsergebnisse spätestens 1 Stunde nach dem Fesseln auf den Wert bei Ruhe zurückkommen; aber die beim Fesseln vermehrte Blutmilchsäure erreicht, wie oben erwähnt, nicht schnell wieder den Wert der Ruhe, was auf den grossen Einfluss der psychischen Aufregung deutet.

B oehm $\mathrm{u}$. Hof $\mathrm{fmann}^{6}$ ) haben bemerkt, dass nach dem Fesseln das Glykogen der Leber und des Muskels verschwindet, Gottschalk u. Pohle, ${ }^{18}$ dass bei Adrenalinhyperglykämie die Wasserstoffionenkonzentration der V. hepatica höher als die der $\mathrm{V}$. portae steigt, und $\mathrm{K}$ a to $\mathrm{u}$. $\mathrm{Kimur}{ }^{19}$ ) aus hiesiger Klinik haben beim Kauinchen, dem Adrenalin injiziert worden ist, nachgewiesen, dass die Zunahme des Blutmilchsäuregehalts der V. heptica grösser ist als die der V. portae. Daraus kann man auch schliessen, dass die Zunahme des Blutzuckers und der Blutmilchsäure beim Fesseln in inniger Beziehung zum Leberglykogen steht.

\section{II. Ửber die Veränderungen des intermediären \\ Kohlehydratstoffwechsels beim \\ Naphthylaminfieber.}

Weil, wie in I. Kapitel beobachtet, die Beeinflussung der Blutmilchsäure des Kaninchens durch Fesseln und Behandeln zurBlutentnahme 2 Stunden danach beinahe aufhört und danach der Milchsäurespiegel nur wenig schwankt, so wurde in den vorliegenden Versuchen 2 Stunden nach dem Fesseln und der Vorbereitung der Blutentnahme das Blut zum ersten Male entnommen und bestimmt, aus dem auf den Blutzuckerund Blutmilchsäuregehalt vor Fieber geschlossen wurde.

16) Feinchemid u. Ferdmann, Bioch. Zeitschr., 1929, 205, 325.

17) Haya s a k a, Tohoku Journ. Exp. Med., 1929-30, 14, 359.

18) Gottschalk u. Pohle, Arch. f. exp. Pathol. u. Pharmakol., 1922, 95, 64.

19) Kato u. Kimura, wird bald in dieser Zeitschrift publiziert. 
Kontrollversuche:-Weil beim eigentlichen Versuche das Fieber durchschnittlich 2 Stunden nach Injektion von Tetrahydro- $\beta$ -

Tabelle 2.

Kontrollversuch zum Naphthylaminfieber-Versuch.

\begin{tabular}{|c|c|c|c|c|c|c|c|c|c|c|}
\hline \multirow{2}{*}{$\mathrm{Nr}$. } & \multirow{2}{*}{$\begin{array}{l}\text { Körper- } \\
\text { gew. u. } \\
\text { Gesch- } \\
\text { lecht }\end{array}$} & \multirow{2}{*}{$\begin{array}{c}\text { Zeit der } \\
\text { Blutentnahme }\end{array}$} & \multicolumn{2}{|c|}{$\begin{array}{l}\text { Körper- } \\
\text { temp. }\end{array}$} & \multirow{2}{*}{ 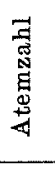 } & \multirow{2}{*}{ 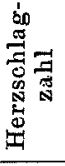 } & \multicolumn{2}{|c|}{$\begin{array}{c}\text { Blutmilch- } \\
\text { säure }\end{array}$} & \multicolumn{2}{|c|}{$\begin{array}{l}\text { Blut- } \\
\text { zucker }\end{array}$} \\
\hline & & & $\mathrm{C}^{\circ}$ & $\begin{array}{c}\text { Diff. } \\
\text { in } \\
\mathrm{C}^{\circ}\end{array}$ & & & $\frac{\vec{g}}{80}$ & $\begin{array}{c}\text { Diff. } \\
\text { in } \\
\%\end{array}$ & $\frac{\vec{Z}}{30}$ & $\begin{array}{c}\text { Diff. } \\
\text { in } \\
\% \\
\end{array}$ \\
\hline 1 & $\begin{array}{c}2070 \mathrm{~g} \\
9\end{array}$ & $\begin{array}{l}\text { Vor der Fesselung } \\
8^{\mathrm{h}} 20^{\prime} \text { a.m. gefesselt } \\
10^{\mathrm{h}} 40^{\prime} \text {." } \\
12 \mathrm{~h} 40^{\prime} \text { p.m. } \\
1^{\mathrm{h}} 40^{\prime} \text { " } \\
2^{\mathrm{h}} 40^{\prime}, \\
3^{\mathrm{h}} 40^{\prime}, "\end{array}$ & $\begin{array}{l}38,6 \\
\\
37,6 \\
38,3 \\
38,5 \\
38,4 \\
38,7\end{array}$ & $\begin{array}{r}+0,7 \\
+0,9 \\
+0,8 \\
+1,1\end{array}$ & & & \begin{tabular}{|l|}
20,57 \\
20,57 \\
19,29 \\
19,29 \\
18,00
\end{tabular} \mid & $\begin{array}{l} \pm 0 \\
-6 \\
-6 \\
-12\end{array}$ & $\begin{array}{l}159 \\
152 \\
127 \\
134 \\
111\end{array}$ & $\begin{array}{l}-4 \\
-20 \\
-16 \\
-30\end{array}$ \\
\hline 2 & $\begin{array}{c}1902 \mathrm{~g} \\
\text { 우 }\end{array}$ & $\begin{array}{l}\text { Vor der Fesselung } \\
8^{\mathrm{h}} 10^{\prime} \text { a.m. gefesselt } \\
10^{\mathrm{l}} 30^{\prime} \text {." } \\
12^{\mathrm{h}} 30^{\prime} \text { p.m. } \\
1^{\mathrm{b}} 30^{\prime}, " \\
2^{\mathrm{h}} 30^{\prime} \text { ", } \\
3^{\mathrm{h}} 30^{\prime}, "\end{array}$ & $\begin{array}{l}39,1 \\
\\
37,5 \\
37,8 \\
38,2 \\
38,5 \\
38,4\end{array}$ & $\begin{array}{l}+0,3 \\
+0,7 \\
+1,0 \\
+0,9\end{array}$ & & & $\begin{array}{l}21,86 \\
19,29 \\
14,14 \\
16,71 \\
19,29\end{array}$ & $\left|\begin{array}{l}-12 \\
-35 \\
-24 \\
-12\end{array}\right|$ & $\begin{array}{l}127 \\
117 \\
132 \\
138 \\
134\end{array}$ & $\begin{array}{r}-8 \\
+4 \\
+9 \\
+6\end{array}$ \\
\hline 3 & $\begin{array}{c}2030 \mathrm{~g} \\
\text { 우 }\end{array}$ & $\begin{array}{l}\text { Vor der Fesselung } \\
4^{\mathrm{h}} 40^{\prime} \text { a.m. gefesselt } \\
7^{\mathrm{h}} \quad " \\
9^{\mathrm{h}} \quad ", \\
10^{\mathrm{h}} \\
11^{\mathrm{h}} \\
12^{\mathrm{h}} \quad \text { p.m. }\end{array}$ & $\begin{array}{l}39,1 \\
\\
37,8 \\
38,4 \\
38,7 \\
38,9 \\
39,1\end{array}$ & $\begin{array}{l}+0,6 \\
+0,9 \\
+1,1 \\
+1,3\end{array}$ & $\begin{array}{l}44 \\
44 \\
48 \\
44 \\
60\end{array}$ & $\begin{array}{l}200 \\
206 \\
212 \\
212 \\
222\end{array}$ & $\left|\begin{array}{l}28,29 \\
27,00 \\
27,00 \\
25,71 \\
29,57\end{array}\right|$ & $\begin{array}{r}-5 \\
-5 \\
-9 \\
+5\end{array}$ & $\begin{array}{l}164 \\
143 \\
141 \\
129 \\
141\end{array}$ & $\begin{array}{l}-13 \\
-14 \\
-21 \\
-14\end{array}$ \\
\hline 4 & $\begin{array}{c}2452 \mathrm{~g} \\
\text { 우 }\end{array}$ & 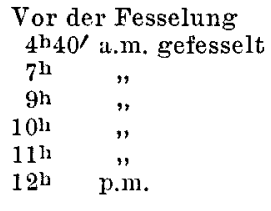 & $\begin{array}{l}39,6 \\
\\
38,4 \\
38,2 \\
38,0 \\
38,7 \\
38,8\end{array}$ & $\begin{array}{l}-0,2 \\
-0,4 \\
+0,3 \\
+0,4\end{array}$ & $\begin{array}{l}52 \\
64 \\
68 \\
68 \\
76\end{array}$ & $\begin{array}{l}248 \\
240 \\
240 \\
232 \\
256\end{array}$ & \begin{tabular}{|l|}
14,14 \\
14,14 \\
14,14 \\
15,43 \\
14,14
\end{tabular} \mid & $\begin{array}{ll} \pm & 0 \\
\pm & 0 \\
+ & 9 \\
\pm & 0\end{array}$ & $\begin{array}{l}122 \\
146 \\
163 \\
148 \\
138\end{array}$ & $\begin{array}{l}+20 \\
+34 \\
+21 \\
+13\end{array}$ \\
\hline 5 & $\begin{array}{c}1940 \mathrm{~g} \\
\widehat{\mathrm{S}}\end{array}$ & $\begin{array}{l}\text { Vor der Fesselung } \\
5^{\mathrm{h}} 10^{\prime} \text { a.m. gefesselt } \\
7^{\mathrm{h}} 30^{\prime} \text { " } \\
9^{\mathrm{h}} 30^{\prime} " \\
10^{\mathrm{h}} 30^{\prime} ", \\
1^{\mathrm{h}} 30^{\prime} \text { " " } \\
12^{\mathrm{h}} 30^{\prime} \text { p.m. }\end{array}$ & $\begin{array}{l}38,7 \\
\\
38,4 \\
38,7 \\
39,0 \\
38,8 \\
38,9\end{array}$ & $\begin{array}{r}+0,3 \\
+0,6 \\
+0,4 \\
+0,5\end{array}$ & $\begin{array}{l}88 \\
90 \\
96 \\
94 \\
96\end{array}$ & $\begin{array}{l}236 \\
240 \\
260 \\
284 \\
276\end{array}$ & $\begin{array}{l}21,86 \\
21,86 \\
23,14 \\
23,14 \\
23,14\end{array}$ & $\begin{array}{l} \pm 0 \\
+6 \\
+6 \\
+6\end{array}$ & $\begin{array}{l}132 \\
119 \\
143 \\
136 \\
138\end{array}$ & $\begin{array}{l}-10 \\
+8 \\
+3 \\
+5\end{array}$ \\
\hline 6 & $\begin{array}{c}2100 \mathrm{~g} \\
\text { 우 }\end{array}$ & 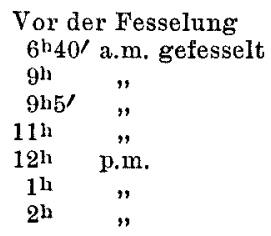 & $\begin{array}{l}38,9 \\
38,8 \\
38,7 \\
38,9 \\
38,9 \\
39,1\end{array}$ & $\begin{array}{l}-\mathrm{T} \text { su } \\
-0,1 \\
+0,1 \\
+0,1 \\
+0,3\end{array}$ & ut & & \begin{tabular}{|l|}
19,29 \\
21,86 \\
19,29 \\
15,43 \\
16,71
\end{tabular} & $\begin{array}{l}+13 \\
\pm 0 \\
-20 \\
-13\end{array}$ & $\begin{array}{l}240 \\
\\
230 \\
190 \\
163 \\
148\end{array}$ & $\begin{array}{r}-4 \\
-21 \\
-32 \\
-38\end{array}$ \\
\hline
\end{tabular}


Fig. د. (Versuch 1, Kontrollversuch zum Naphthylaminfieberversuch.)

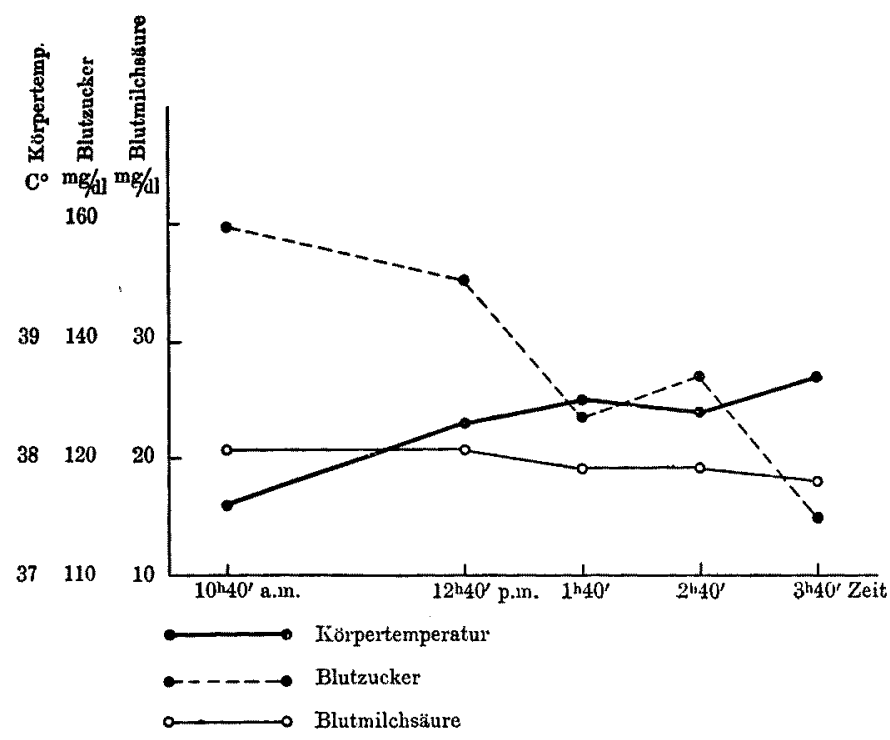

Naphthylaminhydrochlorid (kurz: $\beta$-T.) seinen Höhepunkt erreichte, so wurde auch beim Kontrollversuche zum zweiten Male 2 Stunden nach der ersten Blutentnahme Blut entnommen und danach stündlich, ganz wie beim eigentlichen Versuch, und an diesem wurden Blutzucker und Blutmilchsäure quantitativ bestimmt; die Körpertemperatur bei jeder Blutentnahme wurde auch aufgezeichnet. Hier wurde auch der Fall wie Nr. 6, bei dem nach Injektion von $\beta$-T. keine Körpertemperatursteigerung beobachtet wurde, obwohl Symptome der Sympathikuserregung, wie Mydriasis und Exophthalmus, eintraten, auch mitgerechnet.

Die infolge des Fesselns beträchtlich gesunkene Körpertemperatur wurde allmählich wiederhergestellt und erreichte meist 6 Stunden nach dem Fesseln wieder ihren normalen Wert, bei einigen Fällen aber erholte sie sich selbst dabei noch nicht.

Der Blutzuckerspigel erreichte 2-6 Stunden nach vollendeter Fesselung und Vorbereitung der Blutentnahme den Höhepunkt, danach blieb er beinahe auf derselben Höhe oder zeigte Neigung, allmählich zu sinken; bei Nr. 6, bei dem kein Fieber nach Injektion von $\beta$-T. eintrat, nahm der Blutzucker nach der $\beta$-T-Injektion dauernd ab.

Die Blutmilchsäure schwankte sehr wenig: in der Mehrzahl der Fälle war ihr Gehalt im Zeitpunkt, der beim eigentlichen Versuche dem Stadium des höchsten Fiebers entsprach, d.h. 2 Stunden nach der 
ersten Blutentnahme kleiner als bei der vorangehenden Blutentnahme, und zwar war sie auch bei einem Fall wie Nr. 6, bei dem der Fieberanstieg durch $\beta$-T-Injektion ausblieb, 2 Stunden nach der Injektion ein wenig vermehrt.

Eigentliche Versuche:-Gleich nach der ersten Blutentnahme wurde den Tieren subkutan $5 \%$ ige $\beta$-T-Lösung, $0,5 \mathrm{ccm}$ pro $\mathrm{kg}$ injiziert, und das Blut bei der höchsten Fiebersteigerung zum zwei-

Tabelle 3.

Veränderungen der Blutmilchsäure u. des Blutzuckers

beim Naphthylaminfieber.

\begin{tabular}{|c|c|c|c|c|c|c|c|c|c|c|c|c|}
\hline \multirow[b]{2}{*}{$\dot{\text { z }}$} & \multirow{2}{*}{ 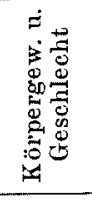 } & \multirow{2}{*}{$\begin{array}{c}\text { Zeit der } \\
\text { Blutentnahme }\end{array}$} & \multicolumn{2}{|c|}{$\begin{array}{c}\text { Körper- } \\
\text { temp. }\end{array}$} & \multirow{2}{*}{ 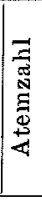 } & \multirow{2}{*}{ 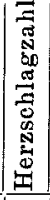 } & \multicolumn{3}{|c|}{ Blutmilchsäure } & \multicolumn{3}{|c|}{ Blutzucker } \\
\hline & & & $\mathrm{C}^{\mathrm{O}}$ & 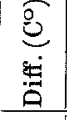 & & & $\frac{F}{300}$ & $\mid \begin{array}{ll}. \\
0 \\
0 \\
6\end{array}$ & 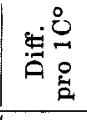 & $\frac{\overrightarrow{0}}{80}$ & $\mid \begin{array}{ll}= \\
0 \\
0\end{array}$ & 造是 \\
\hline 1 & $\begin{array}{c}2000 \mathrm{~g} \\
\delta\end{array}$ & 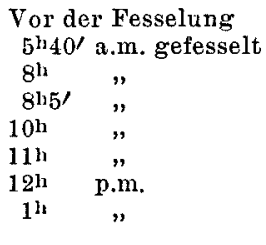 & $\begin{array}{r}39,2 \\
38,2 \\
\beta-T \\
40,6 \\
39,7 \\
39,2 \\
39,3 \\
\end{array}$ & $\begin{array}{r}\text { subk } \\
+2,4 \\
+1,5 \\
+1,0 \\
+1,1\end{array}$ & kuta & & $\begin{array}{l}18,00 \\
28,29 \\
18,00 \\
15,43 \\
14,14\end{array}$ & $\begin{array}{rr}+ & 57 \\
\pm & 0 \\
- & 14 \\
- & 21\end{array}$ & & $\begin{array}{l}181 \\
175 \\
161 \\
177\end{array} \mid$ & $\left|\begin{array}{l}-2 \\
-5 \\
-13 \\
-4\end{array}\right|$ & $-1,25$ \\
\hline 2 & $\begin{array}{c}1863 \mathrm{~g} \\
\hat{\delta}\end{array}$ & $\begin{array}{l}\text { Vor der Fesselung } \\
6^{\mathrm{l}} 40^{\prime} \text { a.m. gefesselt } \\
9^{\mathrm{h}} \text { " } \\
9^{\mathrm{h}} 10^{\prime} \text { " } \\
1^{\mathrm{l}} 20^{\prime} \text { p.m. } \\
2^{\mathrm{l}} 20^{\prime} \text { " ", } \\
3^{\mathrm{h}} 20^{\prime}, " \\
4^{\mathrm{h}} 20^{\prime}, "\end{array}$ & $\begin{array}{r}39,4 \\
39,4 \\
\beta-7 \\
41,9 \\
41,3 \\
40,8 \\
40,6\end{array}$ & $\begin{array}{l}\text { subk } \\
+2,5 \\
+1,9 \\
+1,4 \\
+1,2\end{array}$ & suta & & $\left|\begin{array}{l}28,57 \\
57,80 \\
34,71 \\
37,86 \\
23,14\end{array}\right|$ & $\mid \begin{array}{r}102 \\
+\quad 21 \\
+\quad 8 \\
-\quad 19\end{array}$ & $+11,69$ & $\begin{array}{l}136 \\
124 \\
138 \\
155\end{array}$ & $\begin{array}{l}3 \\
-25 \\
-32 \\
-24 \\
-15\end{array}$ & $-18,40$ \\
\hline 3 & $\begin{array}{c}2030 \mathrm{~g} \\
\delta\end{array}$ & $\begin{array}{l}\text { Vor der Fesselung } \\
6^{\mathrm{l}} 30^{\prime} \text { a.m. gefesselt } \\
8^{\mathrm{l}} 50^{\prime}, " \\
9^{\mathrm{h}} \text { " } \\
10^{\mathrm{h}} 15^{\prime} " \\
11^{\mathrm{h}} 15^{\prime} ", \\
12^{\mathrm{l}} 15^{\prime} \text { p.m. } \\
1^{\mathrm{h}} 15^{\prime}, "\end{array}$ & $\begin{array}{r}39,3 \\
39,0 \\
\beta-T \\
40,2 \\
39,5 \\
39,2 \\
38,8\end{array}$ & $\begin{array}{r}\text { subk } \\
+1,2 \\
+0,5 \\
+0,2 \\
-0,2\end{array}$ & zuta & & \begin{tabular}{|l|}
20,57 \\
\\
38,57 \\
25,71 \\
20,57 \\
20,57
\end{tabular} & $\begin{array}{rr}+ & 88 \\
+ & 25 \\
\pm & 0 \\
\pm & 0\end{array}$ & + & $\begin{array}{l}155 \\
129 \\
129 \\
113\end{array}$ & $\mid \begin{array}{l}+3 \\
-14 \\
-14 \\
-25\end{array}$ & - 4,17 \\
\hline 4 & $\begin{array}{c}1928 \mathrm{~g} \\
\delta\end{array}$ & $\begin{array}{l}\text { Vor der Fesselung } \\
9^{l_{1}} 10^{\prime} \text { a.m. gefesselt } \\
11^{\mathrm{l}} 30^{\prime} \text { " } \\
11^{\mathrm{h}} 35^{\prime} \text { " } \\
1^{\mathrm{h}} \text { p.m. } \\
2^{\mathrm{l}} \quad " \\
3^{\mathrm{l}} \quad " \\
4^{\mathrm{h}} \quad "\end{array}$ & $\mid \begin{array}{r}39,3 \\
38,6 \\
\beta-T \\
40,0 \\
39,8 \\
39,4 \\
39,4\end{array}$ & $\begin{array}{r}\text { subk } \\
+1,4 \\
+1,2 \\
+0,8 \\
+0,8\end{array}$ & cuta & & $\begin{array}{l}18,00 \\
27,00 \\
23,14 \\
18,00 \\
16,71\end{array}$ & $\begin{array}{r}+50 \\
+\quad 29 \\
\pm \quad 0 \\
-\quad 7\end{array}$ & +6 & $\begin{array}{l}193 \\
204 \\
163 \\
117\end{array}$ & $\begin{array}{r}+62 \\
+71 \\
+37 \\
-2\end{array}$ & $+52,86$ \\
\hline
\end{tabular}




\begin{tabular}{|c|c|c|c|c|c|c|c|c|c|c|c|c|}
\hline \multirow[b]{2}{*}{ 离 } & \multirow{2}{*}{ 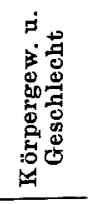 } & \multirow{2}{*}{$\begin{array}{c}\text { Zeit der } \\
\text { Blutentnalime }\end{array}$} & \multicolumn{2}{|c|}{$\begin{array}{c}\text { Körper- } \\
\text { temp. }\end{array}$} & \multirow{2}{*}{  } & \multirow{2}{*}{ 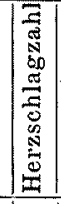 } & \multicolumn{3}{|c|}{ Blutmilchsäure } & \multicolumn{3}{|c|}{ Blutzucker } \\
\hline & & & $\mathrm{C}^{\circ}$ & $\underset{0}{\stackrel{0}{0}}$ & & & $\frac{\text { ह }}{\text { की }}$ & 吾 & $\begin{array}{l}0 \\
0 \\
0 \\
0 \\
0\end{array}$ & 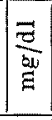 & $\mid \begin{array}{ll}\nexists \\
8 \\
0\end{array}$ & $\begin{array}{l}0 \\
\stackrel{8}{0} \\
0 \\
0\end{array}$ \\
\hline $\mathbf{5}$ & $\begin{array}{c}2270 \mathrm{~g} \\
ㅇ\end{array}$ & $\begin{array}{l}\text { Vor der Fesselung } \\
8^{\mathrm{h} 10^{\prime}} \text { a.m. gefesselt } \\
10^{\mathrm{h}} 30^{\prime}, " \\
10^{\mathrm{h}} 35^{\prime}, " \\
12^{\mathrm{h}} 35^{\prime} \text { p.m. } \\
1^{\mathrm{h} 35^{\prime}}, \\
2^{\mathrm{h}} 35^{\prime} ", \\
3^{\mathrm{b}} 35^{\prime} ",\end{array}$ & $\begin{array}{r}38,8 \\
38,7 \\
\beta-1 \\
40,5 \\
39,3 \\
38,7 \\
38,2\end{array}$ & $\begin{array}{l}\text { sub } \\
+1,8 \\
+0,6 \\
\pm 0 \\
-0,5\end{array}$ & kuta & & $\begin{array}{l}23,14 \\
30,86 \\
30,86 \\
19,29 \\
18,00\end{array}$ & $\begin{array}{r} \\
+\quad 33 \\
+\quad 33 \\
-17 \\
-\quad 22\end{array}$ & & $\begin{array}{l}182 \\
177 \\
146 \\
131\end{array}$ & \begin{tabular}{l|l|}
2 & -14 \\
7 & -16 \\
6 & -31 \\
1 & -38
\end{tabular} & $-16,11$ \\
\hline 6 & $\begin{array}{c}1880 \mathrm{~g} \\
\mathrm{q}\end{array}$ & 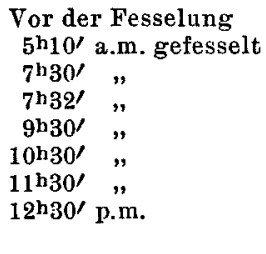 & $\begin{array}{r}38,5 \\
38,2 \\
\beta-1 \\
39,9 \\
39,6 \\
39,4 \\
39,5\end{array}$ & $\begin{array}{l}\text { I subl } \\
+1,7 \\
+1,4 \\
+1,2 \\
+1,3\end{array}$ & $\begin{array}{l}48 \\
\text { kuts } \\
184 \\
140 \\
134 \\
158\end{array}$ & $\begin{array}{l}836 \\
a n \\
4 \\
4276 \\
274 \\
276 \\
266 \\
286\end{array}$ & $\begin{array}{l}19,29 \\
27,00 \\
19,29 \\
19,29 \\
18,00\end{array}$ & $\begin{array}{lr}+ & 40 \\
\pm & 0 \\
\pm & 0 \\
- & 7\end{array}$ & $+4,54$ & $\begin{array}{r}110 \\
97 \\
92 \\
102\end{array}$ & \begin{tabular}{l|l|}
0 & -30 \\
7 & -38 \\
2 & -41 \\
2 & -35
\end{tabular} & $-27,65$ \\
\hline 7 & $\begin{array}{c}1920 \mathrm{~g} \\
\delta\end{array}$ & $\begin{array}{l}\text { Vor der Fesselung } \\
8^{\mathrm{h}} 10^{\prime} \text { a.m. gefesselt } \\
10^{\mathrm{h}} 30^{\prime} \text { " } \\
10^{\mathrm{h}} 35^{\prime} \text {." } \\
12^{\mathrm{h}} 40^{\prime} \text { p.m. } \\
1^{\mathrm{b}} 40^{\prime} \text { " } \\
2^{\mathrm{h}} 40^{\prime} \text { " } \\
3^{\mathrm{h}} 40^{\prime} \text { " }\end{array}$ & $\begin{array}{r}39,5 \\
38,9 \\
\beta- \\
41,0 \\
41,0 \\
40,5 \\
39,9\end{array}$ & $\begin{array}{l}\text { sub } \\
+2,1 \\
+2,1 \\
+1,6 \\
+1,0\end{array}$ & $\begin{array}{r}64 \\
\text { kuts } \\
216 \\
212 \\
162 \\
138\end{array}$ & $\begin{array}{l}4 \mid 210 \\
a^{4} \\
6316 \\
2316 \\
294 \\
268 \\
8 \\
2334\end{array}$ & $\begin{array}{l}19,29 \\
28,29 \\
41,14 \\
34,71 \\
19,29\end{array}$ & $\begin{array}{r}+\quad 47 \\
+113 \\
+\quad 80 \\
\pm \quad 0\end{array}$ & $+4,29$ & $\begin{array}{l}115 \\
120 \\
148 \\
141 \\
111\end{array}$ & $\begin{array}{l}0+4 \\
8+29 \\
1+23 \\
1+3\end{array}$ & $+2,38$ \\
\hline 8 & $\begin{array}{c}2000 \mathrm{~g} \\
\hat{\delta}\end{array}$ & 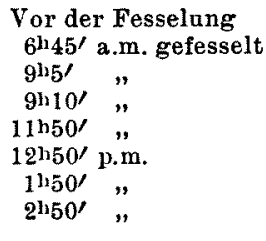 & $\begin{array}{r}39,0 \\
37,7 \\
\beta- \\
40,4 \\
40,1 \\
39,3 \\
38,7\end{array}$ & $\begin{array}{l}\text { sub } \\
+2,7 \\
+2,4 \\
+1,6 \\
+1,0\end{array}$ & $\begin{array}{r}78 \\
\text { kut } \\
241 \\
274 \\
196 \\
94\end{array}$ & \begin{tabular}{l|l}
8 & 232 \\
ian \\
1 \\
1 & 304 \\
4 & 293 \\
6 & 252 \\
4 & 244 \\
\end{tabular} & $\begin{array}{l}30,86 \\
37,29 \\
30,86 \\
30,86 \\
30,86\end{array}$ & $\left|\begin{array}{cc}+ & 21 \\
\pm & 0 \\
\pm & 0 \\
\pm & 0\end{array}\right|$ & & $\begin{array}{l}143 \\
134 \\
129 \\
124\end{array}$ & $\begin{array}{l}3+27 \\
4+19 \\
9+14 \\
4+10\end{array}$ & $+11,11$ \\
\hline 9 & $\begin{array}{c}1900 \mathrm{~g} \\
9\end{array}$ & 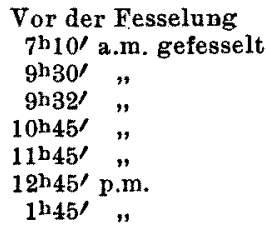 & $\begin{array}{r}38,4 \\
38,1 \\
\beta- \\
40,6 \\
40,4 \\
40,8 \\
40,8\end{array}$ & $\begin{array}{l}\text { sub } \\
+2,5 \\
+2,3 \\
+2,7 \\
+2,7\end{array}$ & $\begin{array}{l}66 \\
\text { kut } \\
5 \mid 130 \\
116 \\
144 \\
138\end{array}$ & \begin{tabular}{l|l|}
6 & 216 \\
aan \\
0 \\
0 \\
6 & 370 \\
4 & 356 \\
828 \\
8 & 348
\end{tabular} & $\begin{array}{l}19,29 \\
50,79 \\
21,22 \\
19,29 \\
21,22\end{array}$ & $\begin{array}{l}9+163 \\
2+10 \\
+\quad 0 \\
\pm+\quad 10\end{array}$ & $+12,60$ & $0 \mid \begin{array}{l}182 \\
240 \\
208 \\
213 \\
208\end{array}$ & $\begin{array}{l}0+32 \\
8+14 \\
3+17 \\
8-14\end{array}$ & $+23,20$ \\
\hline
\end{tabular}

ten Male, danach stündlich während 3 Stunden entnommen, wobei man immer ihre Körpertemperatur aufzeichnete, und an jeder Blutprobe wurden der Blutzucker- und Milchsuäregehalt bestimmt. 
Fig. 3. (Versuch 3, Naphthylaminfieber.)

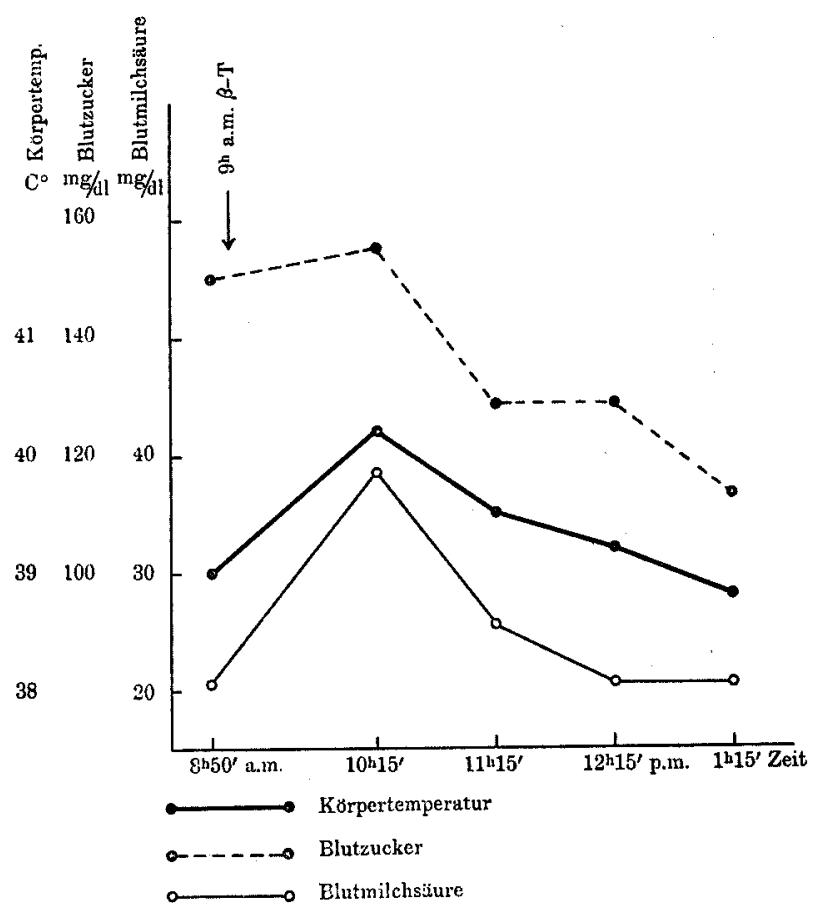

Der Mechanismus der Fiebererzeugung durch Naphthylamin besteht, wie Sachar of $f^{20}$ ) behauptet, darin, dass das Mittel nicht nur durch die Reizung des Wärmezentrums die Wärmebildung fördert, sondern auch durch die Kontraktion der peripheren Blutgefässe die Abgabe der Wärme vermindert; nach dem Fesseln wird, wie er berichtet, manchmal kein Fieber durch $\beta$-T-Injektion erzeugt.

Bei unserem Versuch traten die Symptome der Sympathikuserregung, wie Mydriasis und Exophthalmus, etwa 15 Minuten nach $\beta-\mathrm{T}$ Injektion auf und erreichten nach 30-40 Minuten ihren Höhepunkt. Die durch Fesselung unter die Norm gesunkene Körpertemperatur erhöhte sich erst $\frac{1}{2}-1$ Stunde nach $\beta$-T-Injektion allmählich, wobei sich die Ohrmuschel kalt anfühlten, indem ihre Gefässe zusammenzogen, und erreichte nach $1 \frac{1}{4}-4 \frac{1}{4}$ Stunden den Höhepunkt; danach aber sank die Körpertemperatur allmählich und kam 2-3 Stunden nach dem Erreichen des höchsten Fiebers auf den normalen Wert zurück : nur bei Nr. 9 nahm noch nach 3 Stunden die Körpertemperatur nicht ab; wenn

20) Sacharoff, Zeitschr. f. exp. Pathol. u. Therap, 1910, 7, 225. 
das Fieber dabei über $42^{\circ} \mathrm{C}$ stieg, trat immer der Tod ein, was mit Hasuike $\mathrm{s}^{21}$ Ergebnis übereinstimmt.

Der Blutzuckergehalt nahm beim Fiebern bei einigen Fällen zu, bei den anderen Fällen aber ab, ihre Schwankungen wichen nicht beträchtlich von denen beim Kontrollversuch ab; dies dürfte darauf beruhen, dass der Einfluss des Fesselns auf den Blutzucker so gross ist, dass er die Veränderung des Blutzuckerspiegels durch Fieber iuberschattet.

Trotzdem das $\beta$-T., wie Is en s ch mid ${ }^{22)}$ erklärt, auf die zentralen und peripherischen sympathischen Nerven wirkt, ist die Vermehrung des Blutzuckers doch nicht sehr gross, was schon R os en thal, Licht u. Freu ud d ${ }^{23)}$ berichteten.

Blutmilchsäure nahm beim höchsten Fieber beträchtlich zu und sank 1-2 Stunden danach auf den Wert vor Fieber, was mit der Mitteilung von einem von uns (A d a chi $)^{1)}$ beinahe übereinstimmt. Zwar nahm sie auch bei denjenigen Fällen (z. B. Nr. 6 der Kontrollversuche), in denen die Injektion von $\beta$-T. kein Fieber erzeugen konnte, in der Zeit, wo der grösste Fieberanstieg elwartet wurde, ein wenig zu; aber da ihre Zunahme bei erfolgreicher Injektion viel beträchtlicher ist, so dürfte man wohl annehmen, dass sich die Blutmilchsäure auch infolge des Fiebers selbst vermehrt. Aus der Angabe von Schut, dass Leberglykogen bei Naphthylaminfieber beträchtlich abnimmt, ferner auch aus der Beobachtung von einem von uns (Adachi), ${ }^{1)}$ dass bei Naphthylaminfieber des Hundes trotz der Zunahme der Blutmilchsäure die Milchsäurebildung im Muskel abnimmt, kann man wohl schliessen, dass die Zunahme der Blutmilchsäure auf der gesteigerten Spaltung des Leberglykogens beruht. Die Zunahme der Blutmilchsäure infolge des Fiebers betrug pro $1^{\circ} \mathrm{C} 2,38 \%-15,0 \%$; diese grosse Variabilität des Vermehrungsprozentsatzes könnte auch von dem Leberglykogengehalt des Tiers abhängen. Embden u. $\mathrm{Kraus}^{25)}$ und Oppenheimer ${ }^{26}$ berichten, dass die Milchsäurebildung beim Durchbluten der glykogenarmen Leber geringer ist als bei dem der glykogenreichen Leber.

III. Über die Veränderungen des intermediären Kohlehydratstoffwechsels beim Wärmestichfieber.

Kontrollversuch:-Diejenigen Versuchsfälle, bei denen der

21) Hasuike, Okayama Igakkai Zasshi, 1928, 40, 1725.

22) Isensch mid, Münch. med. Wochenschr., 1914, 1756. 103,17

23) Rosenthal, Licht u. Freund, Arch. f. exp. Pathol. u. Pharmakol., 1924,

24) Schut, Beitıäge z. Kl. d. Tuberkulose, 1916, 35, 75

25) Embden u. Kraus, Bioch. Zeitschr. 1912, 45, 1 ,

26) Oppenheimer, Bioch. Zeitschr., 1912, 45, 30. 
Wärmestich, welcher unter denselben Bedingungen wie beim eigentl1chen Versuche ausgeführt wurde (s.u.), selbst nach 5 Stunden (d.h. der durchschnittlichen Zeit bis zum Erreichen des höchsten Fiebers nach dem Wärmestich beim eigentlichen Versuche) erfolglos war, also keine Körpertemperaturesteigerung bewirkte, diente als die Kontrollver-

Tabelle 4.

Kontrollversuch zum Wärmestichfieber-Versuch.

\begin{tabular}{|c|c|c|c|c|c|c|c|c|c|c|}
\hline \multirow{2}{*}{$\mathrm{Nr}$. } & \multirow{2}{*}{$\begin{array}{c}\text { Körper- } \\
\text { gew. u. } \\
\text { Gesch- } \\
\text { lecht }\end{array}$} & \multirow{2}{*}{$\begin{array}{c}\text { Zeit der } \\
\text { Blutentuahme }\end{array}$} & \multicolumn{2}{|c|}{$\begin{array}{c}\text { Körper- } \\
\text { temp. }\end{array}$} & \multirow{2}{*}{ 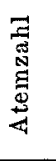 } & \multirow{2}{*}{ 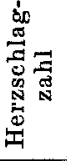 } & \multicolumn{2}{|c|}{$\begin{array}{l}\text { Blutmilch- } \\
\text { säure }\end{array}$} & \multicolumn{2}{|c|}{$\begin{array}{l}\text { Blut- } \\
\text { zucker }\end{array}$} \\
\hline & & & $\mathbf{C}^{\circ}$ & $\begin{array}{c}\text { Ditf. } \\
\text { in } \\
\mathrm{C}^{\circ}\end{array}$ & & & $\frac{7}{800}$ & $\begin{array}{c}\text { Ditf. } \\
\text { in } \\
\%\end{array}$ & $\frac{7}{80}$ & $\begin{array}{c}\text { Diff. } \\
\text { in } \\
\% \\
\end{array}$ \\
\hline 1 & $\begin{array}{c}2500 \mathrm{~g} \\
\text { 웅 }\end{array}$ & $\begin{array}{l}\text { Vor der Fesselung } \\
6^{\mathrm{h}} 10^{\prime} \text { a.m. gefesselt } \\
8^{\mathrm{h}} 30^{\prime}, \\
8^{\mathrm{h}} 45^{\prime}, \\
1^{\mathrm{l}} 45^{\prime} \text { p.m. } \\
2^{\mathrm{l}} 45^{\prime} \text {." } \\
3^{\mathrm{h}} 45^{\prime}, " \\
4^{\mathrm{l}} 45^{\prime}, " \\
5^{\mathrm{h}} 45^{\prime}, "\end{array}$ & $\begin{array}{l}38,7 \\
38,5 \\
38,8 \\
38,9 \\
38,9 \\
38,8 \\
38,8\end{array}$ & $\begin{array}{l}\text { Wär'm } \\
+0,3 \\
+0,4 \\
+0,4 \\
+0,3 \\
+0,3\end{array}$ & $\begin{array}{c}76 \\
\text { stich } \\
34 \\
34 \\
38 \\
40 \\
40\end{array}$ & $\begin{array}{l}254 \\
252 \\
250 \\
262 \\
266\end{array}$ & $\begin{array}{l}24,43 \\
22,51 \\
23,79 \\
23,79 \\
25,08 \\
23,14\end{array}$ & $\begin{array}{r}-8 \\
-3 \\
-\quad 3 \\
+\quad 3 \\
-5\end{array}$ & $\begin{array}{l}152 \\
\\
132 \\
125 \\
125 \\
127 \\
122\end{array}$ & $\begin{array}{l}-13 \\
-18 \\
-18 \\
-16 \\
-20\end{array}$ \\
\hline 2 & $\begin{array}{c}2200 \mathrm{~g} \\
\delta\end{array}$ & 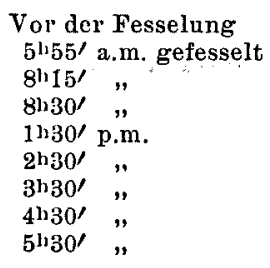 & $\begin{array}{l}38,4 \\
37,2 \\
37,5 \\
37,5 \\
37,5 \\
37,5 \\
37,5\end{array}$ & $\begin{array}{l}\text { Wärm } \\
\mid \begin{array}{l}+0,3 \\
+0,3 \\
+0,3 \\
+0,3 \\
+0,3\end{array}\end{array}$ & $\begin{array}{c}42 \\
\text { stich } \\
30 \\
30 \\
30 \\
32 \\
36\end{array}$ & $\begin{array}{l}200 \\
200 \\
208 \\
218 \\
224\end{array}$ & $\begin{array}{l}19,29 \\
19,94 \\
19,29 \\
18,00 \\
18,00 \\
18,65\end{array}$ & $\begin{array}{l}+3 \\
\pm 0 \\
-7 \\
-\quad 7 \\
-3\end{array}$ & $\begin{array}{l}120 \\
119 \\
117 \\
119 \\
117 \\
117\end{array}$ & $\begin{array}{l}-1 \\
-3 \\
-1 \\
-3 \\
-3\end{array}$ \\
\hline 3 & $\begin{array}{c}2330 \mathrm{~g} \\
\mathrm{q}\end{array}$ & 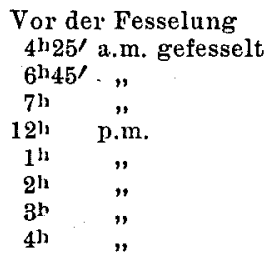 & $\begin{array}{l}39,3 \\
37,7 \\
38,3 \\
38,3 \\
38,4 \\
38,4 \\
38,4\end{array}$ & $\begin{array}{l}\text { Wärm } \\
+0,6 \\
+0,6 \\
+0,7 \\
+0,7 \\
+0,7\end{array}$ & $\begin{array}{c}64 \\
\text { stich } \\
52 \\
50 \\
60 \\
54 \\
58\end{array}$ & $\begin{array}{l}226 \\
228 \\
220 \\
220 \\
222\end{array}$ & $\begin{array}{l}18,65 \\
17,36 \\
19,29 \\
18,65 \\
18,65 \\
16,71\end{array}$ & $\begin{array}{r}-7 \\
+3 \\
\pm 0 \\
\pm 0 \\
-10\end{array}$ & $\begin{array}{l}177 \\
\\
120 \\
117 \\
115 \\
115 \\
115\end{array}$ & $\begin{array}{l}-32 \\
-34 \\
-35 \\
-35 \\
-35\end{array}$ \\
\hline 4 & $\begin{array}{c}2350 \mathrm{~g} \\
\text { q }\end{array}$ & $\begin{array}{l}\text { Vor der Fesselung } \\
4^{\mathrm{l}} 40^{\prime} \text { a.m. gefesselt } \\
7^{\mathrm{h}} \text { ", } \\
7^{\mathrm{h}} 15^{\prime} " \\
12^{\mathrm{h}} 15^{\prime} \text { p.m. } \\
1^{\mathrm{h}} 15^{\prime} \text { " } \\
2^{\mathrm{h}} 15^{\prime} " \\
3^{\mathrm{h}} 15^{\prime} ", \\
4^{\mathrm{h}} 15^{\prime} ",\end{array}$ & $\begin{array}{l}39,3 \\
38,0 \\
38,2 \\
38,8 \\
38,7 \\
38,8 \\
38,9\end{array}$ & $\begin{array}{l}\text { Wärm } \\
\begin{array}{l}+0,2 \\
+0,8 \\
+0,7 \\
+0,8 \\
+0,9\end{array}\end{array}$ & $\begin{array}{c}64 \\
\text { stich } \\
52 \\
50 \\
58 \\
58 \\
50\end{array}$ & $\begin{array}{l}252 \\
258 \\
256 \\
254 \\
256\end{array}$ & $\begin{array}{l}18,65 \\
17,36 \\
18,65 \\
18,00 \\
18,65 \\
19,94\end{array}$ & $\begin{array}{r}-7 \\
\pm \quad 0 \\
-3 \\
\pm \quad \\
+\quad 7\end{array}$ & $\begin{array}{l}127 \\
124 \\
125 \\
119 \\
119 \\
120\end{array}$ & $\begin{array}{r}-2 \\
-2 \\
-6 \\
-6 \\
-6\end{array}$ \\
\hline
\end{tabular}




\begin{tabular}{|c|c|c|c|c|c|c|c|c|c|c|}
\hline \multirow{2}{*}{ Nr. } & \multirow{2}{*}{$\begin{array}{l}\text { Körper- } \\
\text { gew, a. } \\
\text { Gesch- } \\
\text { lecht }\end{array}$} & \multirow{2}{*}{$\begin{array}{c}\text { Zeit der } \\
\text { Blutentnahme }\end{array}$} & \multicolumn{2}{|c|}{$\begin{array}{l}\text { Körper* } \\
\text { temp. }\end{array}$} & \multirow{2}{*}{ 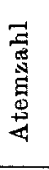 } & \multirow{2}{*}{  } & \multicolumn{2}{|c|}{$\begin{array}{c}\text { Blutmilch- } \\
\text { säure }\end{array}$} & \multicolumn{2}{|c|}{$\begin{array}{l}\text { Blut- } \\
\text { zucker }\end{array}$} \\
\hline & & & $\mathrm{C}^{\circ}$ & $\begin{array}{c}\text { Diff. } \\
\text { in } \\
\mathrm{C}^{\circ} \\
\end{array}$ & & & $\frac{F}{80}$ & $\begin{array}{c}\text { Diff. } \\
\text { in } \\
\%\end{array}$ & $\frac{\vec{B}}{80}$ & $\begin{array}{c}\text { Diti. } \\
\text { in } \\
\%\end{array}$ \\
\hline $\boldsymbol{5}$ & $\begin{array}{c}2060 \mathrm{~g} \\
\hat{\delta}\end{array}$ & 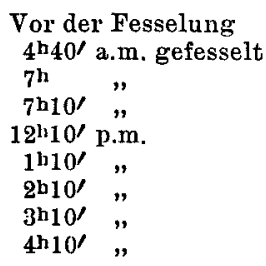 & $\begin{array}{l}39,1 \\
36,8 \\
\\
37,3 \\
37,4 \\
37,4 \\
37,2 \\
37,3\end{array}$ & $\begin{array}{l}\text { Wäm } \\
+0,5 \\
+0,6 \\
+0,6 \\
+0,4 \\
+0,5\end{array}$ & $\begin{array}{l}62 \\
\text { stich } \\
68 \\
64 \\
60 \\
60 \\
60\end{array}$ & $\begin{array}{l}256 \\
258 \\
260 \\
264 \\
280\end{array}$ & $\begin{array}{l}12,86 \\
11,57 \\
12,22 \\
12,92 \\
14,14 \\
12,22\end{array}$ & $\begin{array}{r}-10 \\
-5 \\
-5 \\
+10 \\
-5\end{array}$ & $\begin{array}{l}181 \\
\\
145 \\
145 \\
145 \\
145 \\
146\end{array}$ & $\begin{array}{l}-20 \\
-20 \\
-20 \\
-20 \\
-19\end{array}$ \\
\hline 6 & $\begin{array}{c}2050 \mathrm{~g} \\
\text { ㅇ }\end{array}$ & 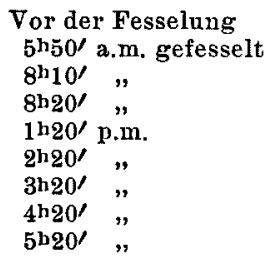 & $\begin{array}{l}39,5 \\
\\
39,0 \\
\\
39,5 \\
39,5 \\
39,5 \\
39,6 \\
39,3\end{array}$ & $\begin{array}{l}\text { Wärm } \\
\qquad \begin{array}{l}+0,5 \\
+0,5 \\
+0,5 \\
+0,6 \\
+0,3\end{array}\end{array}$ & $\begin{array}{c}66 \\
\text { sticl } \\
50 \\
42 \\
54 \\
42 \\
44\end{array}$ & $\begin{array}{l}212 \\
242 \\
260 \\
250 \\
256\end{array}$ & $\begin{array}{l}18,65 \\
18,00 \\
18,65 \\
18,00 \\
18,00 \\
17,36\end{array}$ & $\begin{array}{rr}- & 3 \\
\pm & 0 \\
- & 3 \\
- & 3 \\
- & 7\end{array}$ & $\begin{array}{r}108 \\
\\
110 \\
110 \\
102 \\
97 \\
108\end{array}$ & $\begin{array}{l}+2 \\
+\quad 2 \\
-6 \\
-10 \\
\pm 0\end{array}$ \\
\hline
\end{tabular}

Fig. 4. (Versuch 3, Kontrollversuch zum Wärmestichfieberversuch.)

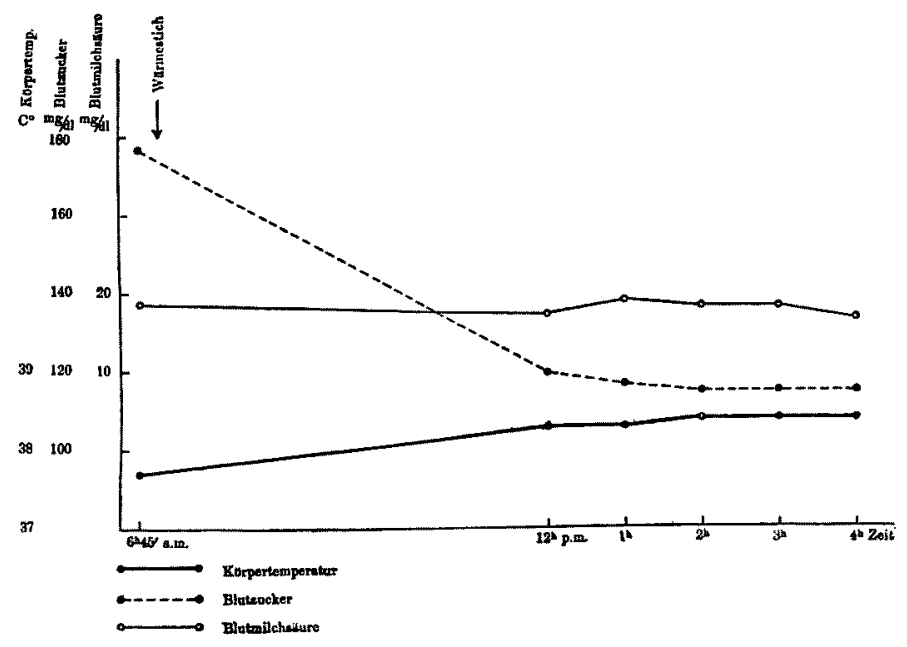

suche für die Feststellung, ob beim mechanischen Reiz des Wärmezentrums die Veränderung des Kohlehydratstoffwechsels der Körpertemperatur' steigerung zuzuschreiben ist, weil bei unserem Versuch der Stich 
des Corpus striatum den verschiedenen Stoffwechselzentren, die im $Z$ wischenhirn vorhanden sind, auch manchen Anreiz geben kann.

Die bei der ersten Blutentnahme unter die Norm gesunkene Körpertemperatur konnte meist sogar 5 Stunden nach dem Wärmestich die normale Höhe noch nicht erreichen, und danach war während 4 Stunden kaum Körpertemperaturveränderung zu beobachten. Die Atemfrequenz nahm im allgemeinen 5 Stunden nach dem Wärmestich ab, was beweist, dass das Tier infolge der Fesselung einmal aufgeregt worden waren, sich jedoch danach allmählich wieder beruhigt hatten; die Pulszahl vermehrte sich allmählich gegen Ende des Versuchs, wahrscheinlich weil infolge der langen Fesselung und der häufigen Blutentnahme mehr oder weniger starke Herzschwäche eintrat.

Der infolge der Fesselung vermehrte Blutzucker verminderte sich im allgemeinen 5 Stunden nach erfolglosem Wärmestich bis auf 110$145 \mathrm{mg} / \mathrm{dl}$, dann aber war die Schwankung des Blutzuckerwerts während 4 Stunden sehr klein.

Der Blutmilchsäuregehalt veründerte sich kaum weder vor noch nach dem erfolglosen Wärmestich.

Eigentliche Versuche:-

Rolly, ${ }^{27)}$ Seuator u. Richter ${ }^{28)}$ berichte ten, dass beim Tier, dessen Leberglykogen vermindert ist, der Wärmestich kein oder nur leichtes Fieber er-

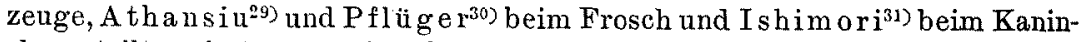
chen stellten fest, dass die Ablagerung von Leberglykogen im Sommer am kleinsten ist. Deshalb machten wir den betreffenden Versuch vom Ende des Herbstes bis zum Anfang des nächsten Frühlings an wenigstens 3 Wochen lang mit dem oben erwähnten Futter gut ernährten Kaninchen.

Was die Versuchsanordnung betrifft, so wurden die Kaninchen, wie oben erwähnt, zuerst auf dem Tierhalter gefesselt und gleich nach der ersten Blutentnahme wurde der Wärmestich ausgeführt und beim Erreichen des höchsten Fiebers zum zweiten Male Blut entnommen, danach aber stündlich während 4 Stunden, wobei jeweils die Körpertemperatur im Rectum gemessen wurde. Der Zucker- und Milchsäuregehalt jeder Blutprobe des dabei entnommenen Bluts wurde quantitativ bestimmt. Der Wärmestich wurde nach der Aronsohn u. Sachsschen ${ }^{32}$ Methode ausgeführt.

Die Körpertemperatur erreichte den Höhepunkt $\left(39,9-41,8^{\circ} \mathrm{C}\right)$ $3 \frac{1}{4}-7 \frac{1}{4}$ Stunden, durchschnittlich 5 Stunden nach Wärmestich, blieb aber dann während 4 Stunden fast auf demselben hohen Grade.

27) Rolly, Dtsch. Alch. f. kl. Med., 1903, 78, 250.

28) Senator u. Richter, Zeitschr. f. kI. Med., 1904, 54, 16.

29) Athanasiu, Pflüger's Arch, 1899, 74, 561.

30) Pflüger, Pflüger's Arch, 1907, 120, 253.

31) Ishim ori, Chuo Igakkai Zasshi, 1913, 112, 1.

32) Aronsohn u. Sachs, Pflüger's Areh., 1885, 37, 232. 
Tabelle 5.

Veränderungen der Blutmilchsäure u. des Blutzuckers beim Wärmstichfieber.

\begin{tabular}{|c|c|c|c|c|c|c|c|c|c|c|c|c|}
\hline \multirow[b]{2}{*}{ 占 } & \multirow{2}{*}{ 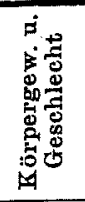 } & \multirow{2}{*}{$\begin{array}{c}\text { Zeit der } \\
\text { Blutentnahme }\end{array}$} & \multicolumn{2}{|c|}{$\begin{array}{c}\text { Körper- } \\
\text { temp. }\end{array}$} & \multirow{2}{*}{ 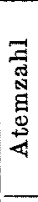 } & \multirow{2}{*}{  } & \multicolumn{3}{|c|}{ Blutmilchsäure } & \multicolumn{3}{|c|}{ Blutzncker } \\
\hline & & & $\mathrm{C}^{\circ}$ & 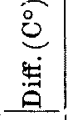 & & & $\frac{\Xi}{80}$ & $\mid \begin{array}{ll}a \\
0 \\
0\end{array}$ & 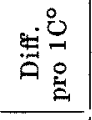 & $\frac{\overrightarrow{0}}{80}$ & $\left|\begin{array}{ll}a & \\
4 & 0 \\
0 & 0\end{array}\right|$ & $\begin{array}{l}0 \\
0 \\
0 \\
0 \\
0 \\
0 \\
0\end{array}$ \\
\hline 1 & $\begin{array}{c}2520 \mathrm{~g} \\
\text { ㅇ }\end{array}$ & 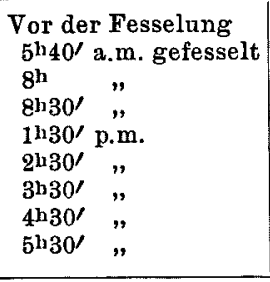 & $\begin{array}{r}39,2 \\
38,3 \\
W \\
40,9 \\
41,0 \\
40,8 \\
40,8 \\
40,8\end{array}$ & $\begin{array}{l}\text { ärmes } \\
+2,6 \\
+2,5 \\
+2,5 \\
+2,5 \\
+2,5\end{array}$ & $\begin{array}{r}54 \\
\text { stic } \\
72 \\
72 \\
68 \\
64 \\
68\end{array}$ & $\begin{array}{l}240 \\
2 \\
252 \\
278 \\
276 \\
276 \\
276 \\
268\end{array}$ & $\begin{array}{l}32,14 \\
36,00 \\
45,00 \\
34,71 \\
33,43 \\
30,86\end{array}$ & $\begin{array}{r}+12 \\
+40 \\
+\quad 8 \\
+\quad 4 \\
-\quad 4\end{array}$ & $+1,48$ & $\begin{array}{l}152 \\
145 \\
141 \\
145 \\
152\end{array}$ & $\left|\begin{array}{l}-23 \\
-26 \\
-28 \\
-26 \\
-23\end{array}\right|$ &, 31 \\
\hline 2 & $\begin{array}{c}2120 \mathrm{~g} \\
\text { 우 }\end{array}$ &  & $\begin{array}{r}39,3 \\
38,8 \\
\text { W } \\
40,2 \\
40,0 \\
40,3 \\
40,3 \\
40,2\end{array}$ & $\begin{array}{r} \\
+1,4 \\
+1,2 \\
+1,5 \\
+1,5 \\
+1,4\end{array}$ & $\begin{array}{l}67 \\
\text { stic } \\
124 \\
148 \\
128 \\
128 \\
120\end{array}$ & $\begin{array}{l}7 \mid 216 \\
6 h \\
4244 \\
8260 \\
8240 \\
8252 \\
0240\end{array}$ & $\begin{array}{l}32,14 \\
57,86 \\
48,86 \\
57,86 \\
32,14 \\
29,57\end{array}$ & $\begin{array}{l}+80 \\
+52 \\
+80 \\
\pm 0 \\
-8\end{array}$ & $+18,37$ & $\begin{array}{l}200 \\
255 \\
247 \\
199 \\
154 \\
150\end{array}$ & $\left|\begin{array}{r}+28 \\
+24 \\
-1 \\
-23 \\
-25\end{array}\right|$ & $+39,29$ \\
\hline 3 & $\begin{array}{c}2280 \mathrm{~g} \\
\text { 우 }\end{array}$ & $\begin{array}{l}\text { Vor der Fesselung } \\
3^{\text {h } 20^{\prime}} \text { a.m. gefesselt } \\
5^{\text {h } 40^{\prime}} \text { " } \\
5^{\text {h }} 0^{\prime} \text { ", } \\
1^{\mathrm{h}} \quad \text { p.m. } \\
2^{\mathrm{h}} \quad " \\
3^{\mathrm{h}} \quad " \\
4^{\mathrm{h}} \quad "\end{array}$ & \begin{tabular}{r|}
39,1 \\
38,2 \\
$W$ \\
40,1 \\
39,8 \\
39,8 \\
39,8
\end{tabular} & $\begin{array}{l}\text { ärme } \\
+1,9 \\
+1,6 \\
+1,6 \\
+1,6\end{array}$ & $\begin{array}{r}60 \\
\text { stic } \\
112 \\
96 \\
96 \\
84\end{array}$ & \begin{tabular}{l|l|}
0 & 216 \\
$\mathrm{ch}$ \\
2 \\
2 \\
6226 \\
2224 \\
6 & 276 \\
4 & 270
\end{tabular} & $\begin{array}{l}25,08 \\
32,14 \\
27,65 \\
25,08 \\
25,71\end{array}$ & $\begin{array}{l}+28 \\
+10 \\
\pm \\
+\quad 3\end{array}$ & $+3,72$ & $\begin{array}{l}121 \\
138 \\
136 \\
121 \\
139\end{array}$ & $\left|\begin{array}{rr}+14 \\
+12 \\
\pm 0 \\
+15\end{array}\right|$ & +8 \\
\hline 4 & $\begin{array}{c}2130 \mathrm{~g} \\
\delta\end{array}$ & $\begin{array}{l}\text { Vor der Fesselung } \\
6^{\mathrm{h}} \text { a.m. gefesselt } \\
8^{\mathrm{h}} 20^{\prime} \text { " } \\
8^{\mathrm{h}} 45^{\prime} \text { " } \\
12 \mathrm{~h} \quad \text { p.m. } \\
1^{\mathrm{h}} \quad " \\
2^{\mathrm{h}} \quad " \\
3^{\mathrm{h}} \quad " \\
4^{\mathrm{h}} \quad "\end{array}$ & $\begin{array}{r}39,4 \\
38,1 \\
W \\
41,8 \\
41,9 \\
41,8 \\
41,9 \\
41,9\end{array}$ & $\begin{array}{l} \\
\text { ärme } \\
+3,7 \\
+3,8 \\
+3,7 \\
+3,8 \\
+3,8\end{array}$ & $\begin{array}{r}72 \\
\text { stic } \\
144 \\
150\end{array}$ & $\begin{array}{ll}2 & 228 \\
\mathrm{ch} & \\
4 & 360 \\
0 & 360 \\
0 & 320 \\
0 & 326 \\
5 & 306\end{array}$ & $\begin{array}{l}21,86 \\
33,43 \\
29,57 \\
24,43 \\
21,22 \\
21,86\end{array}$ & $\begin{array}{l}+53 \\
+35 \\
3+12 \\
-3 \\
-5\end{array}$ & $+3,13$ & $\begin{array}{l}161 \\
188 \\
193 \\
184 \\
195\end{array}$ & $\begin{array}{l}+30 \\
+52 \\
+56 \\
+48 \\
+57\end{array}$ & +1 \\
\hline $\mathbf{5}$ & $\begin{array}{c}2100 \mathrm{~g} \\
\text { 우 }\end{array}$ & $\begin{array}{l}\text { Vor der Fesselung } \\
5^{\mathrm{h}} 10^{\prime} \text { a.m. gefesselt } \\
7^{\mathrm{h}} 30^{\prime}, " \\
8^{\mathrm{h}} \text { " } \\
1^{\mathrm{h}} 50^{\prime} \text { p.m. } \\
2^{\mathrm{h}} 50^{\prime}, " \\
3^{\mathrm{h}} 50^{\prime}, " \\
4^{\mathrm{b}} 50^{\prime} " \\
5^{\mathrm{h}} 50^{\prime} ",\end{array}$ & $\begin{array}{r}39,0 \\
\\
38,3 \\
W \\
41,1 \\
40,9 \\
41,2 \\
41,0 \\
41,3\end{array}$ & $\begin{array}{l}\text { ärme } \\
+2,8 \\
+2,6 \\
+2,9 \\
+2,7 \\
+3,0\end{array}$ & $\begin{array}{l}64 \\
84 \\
84 \\
85 \\
84 \\
80\end{array}$ & \begin{tabular}{l|l|}
4 & 240 \\
$\mathrm{ch}$ & \\
4 & 332 \\
4 & 324 \\
5 & 330 \\
4 & 336 \\
0 & 332
\end{tabular} & $\begin{array}{l}21,86 \\
28,94 \\
25,08 \\
26,46 \\
20,57 \\
22,51\end{array}$ & $\begin{array}{l}4 \\
3+32 \\
6 \\
6+21 \\
7 \\
1+3 \\
+\quad 3\end{array}$ & $+2,53$ & $\begin{array}{l}191 \\
234 \\
247 \\
251 \\
202\end{array}$ & $\begin{array}{r}+15 \\
+41 \\
+49 \\
+51 \\
+22\end{array}$ & $+8,93$ \\
\hline
\end{tabular}




\begin{tabular}{|c|c|c|c|c|c|c|c|c|c|c|c|c|}
\hline \multirow[b]{2}{*}{$\dot{\vec{z}}$} & \multirow{2}{*}{ 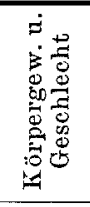 } & \multirow{2}{*}{$\begin{array}{c}\text { Zeit der } \\
\text { B]utentnahme }\end{array}$} & \multicolumn{2}{|c|}{$\begin{array}{l}\text { liörper- } \\
\text { temp. }\end{array}$} & \multirow{2}{*}{ 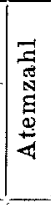 } & \multirow{2}{*}{ 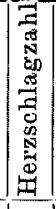 } & \multicolumn{3}{|c|}{ Blutmilchsäure } & \multicolumn{3}{|c|}{ Blutzucker } \\
\hline & & & $\mathrm{C}^{\circ}$ & 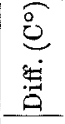 & & & $\frac{\vec{z}}{\text { कृ }}$ & 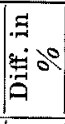 & $\begin{array}{l}0 \\
0 \\
0 \\
0 \\
0 \\
0\end{array}$ & $\frac{\frac{F}{\partial 0}}{\partial 0}$ & $\mid \begin{array}{ll}z \\
0 \\
0\end{array}$ & 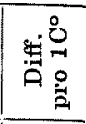 \\
\hline 6 & $\begin{array}{c}2000 \mathrm{~g} \\
\text { 우 }\end{array}$ & $\begin{array}{l}\text { Vor der Fesselung } \\
6^{\mathrm{h}} 40^{\prime} \text { a.m. gefesselt } \\
9^{\mathrm{h}} \quad, \\
9^{\mathrm{h}} 15^{\prime}, " \\
12^{\mathrm{h}} 45^{\prime} \text { p.m. } \\
1^{\mathrm{h}} 45^{\prime}, ", \\
2^{\mathrm{h}} 45^{\prime}, " \\
3^{\mathrm{h}} 45^{\prime}, " \\
4^{\mathrm{h}} 45^{\prime}, "\end{array}$ & $\begin{array}{r}39,0 \\
38,0 \\
W \\
39,9 \\
39,9 \\
40,0 \\
40,0 \\
39,9\end{array}$ & $\begin{array}{r} \\
\text { ärme } \\
+1,9 \\
+1,9 \\
+2,0 \\
+2,0 \\
+1,9\end{array}$ & $\begin{array}{l}78 \\
\text { stic } \\
112 \\
106 \\
108^{\prime} \\
114^{\prime} \\
118^{\prime}\end{array}$ & $\begin{array}{l}228 \\
\mathrm{~h} \\
300 \\
285 \\
292 \\
302 \\
310\end{array}$ & $\begin{array}{l}27,00 \\
37,29 \\
33,43 \\
34,71 \\
30,86 \\
26,62\end{array}$ & $\begin{array}{l}+38 \\
+24 \\
+29 \\
+14 \\
-1\end{array}$ & $+5,42$ & $\begin{array}{l}130 \\
197 \\
154 \\
138 \\
132 \\
139\end{array}$ & $\begin{array}{r}+52 \\
+18 \\
+\quad 6 \\
+\quad 2 \\
+\quad 7\end{array}$ & $+35,26$ \\
\hline 7 & $\begin{array}{c}2300 \mathrm{~g} \\
\mathrm{f}\end{array}$ & $\begin{array}{l}\text { Vor der Fesselung } \\
7^{\mathrm{h}} 40^{\prime} \text { a.m. gefesselt } \\
10^{\mathrm{h}}, \\
10^{\mathrm{h}} 15^{\prime}, " \\
2^{\mathrm{h}} 30^{\prime} \text { p.m. } \\
3^{\mathrm{l}} 30^{\prime}, " \\
4^{\mathrm{h}} 30^{\prime}, " \\
5^{\mathrm{h}} 30^{\prime}, " \\
6^{\mathrm{l}} 30^{\prime}, "\end{array}$ & $\begin{array}{r}38,5 \\
W \\
40,0 \\
39,8 \\
40,2 \\
39,9 \\
40,0\end{array}$ & $\begin{array}{l} \\
\text { ärme } \\
+1,5 \\
+1,3 \\
+1,7 \\
+1,4 \\
+1,5\end{array}$ & $\begin{array}{r}64 \\
\text { sticl } \\
120 \\
120 \\
106 \\
88 \\
92\end{array}$ & $\begin{array}{l}180 \\
h \\
220 \\
252 \\
260 \\
238 \\
258\end{array}$ & $\begin{array}{l}20,57 \\
26,46 \\
36,00 \\
30,22 \\
21,86 \\
23,79\end{array}$ & $\begin{array}{r}+29 \\
+75 \\
+47 \\
+6 \\
+16\end{array}$ & $+3,93$ & $\begin{array}{l}119 \\
124 \\
139 \\
150 \\
134 \\
145\end{array}$ & $\begin{array}{r}+4 \\
+17 \\
+26 \\
+13 \\
+22\end{array}$ & $+3,33$ \\
\hline
\end{tabular}

Fig. 5. (Versuch 2, Wärmestichfieber.)

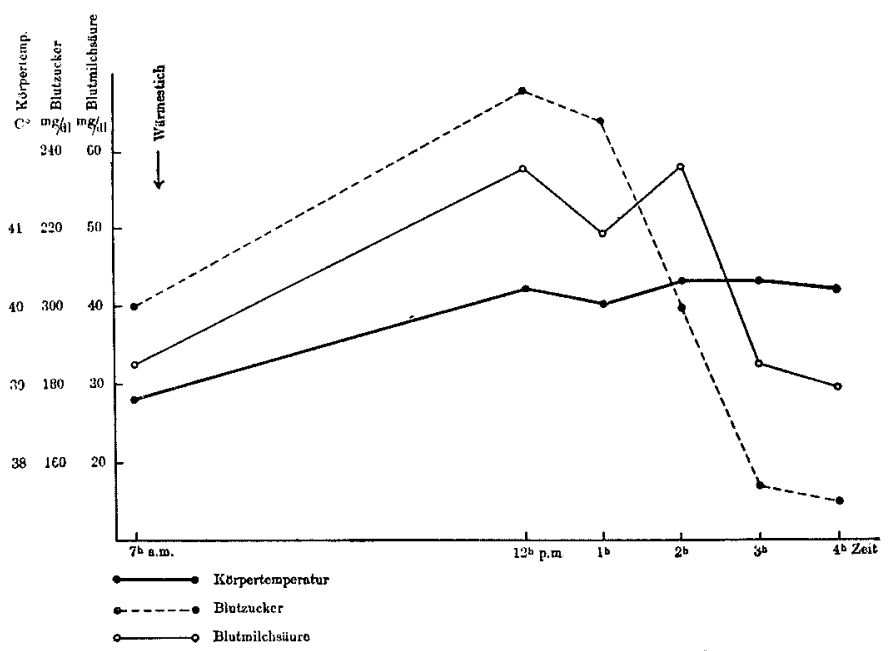

Aronsohn u. Sachs ${ }^{32)}$ stellten bei Wärmestich am gefesselten Kaninchen fest, dass die Körpertemperatur langsamer steigt als beim nicht gefesselten und sich meist nach 5 Stunden zum höchsten Punkt erhöht, der auch niedriger ist als beim frei gelassenen Tier. 
Atem- und Pulszahl vermehren sich nach dem Fieber beträchttich.

Aronsohn u. Sachs ${ }^{32}$ ) haben Zunahme des $\mathrm{O}_{2}$-Verbrauchs und der $\mathrm{CO}_{2}$ Ausscheidung bei Wärmestichfieber bemerkt, Odaira ${ }^{33}$ dabei Zunahme des Grundumsatzes und des Herz-Minutenvolums; Schultz $\mathrm{e}^{3+}$ ) hat nachgewiesen, dass im Stadium des steigenden Fiebers durch Wärmestich die Wärmebildung erheblich, aber die Wärmeabgabe wenig zunimmt, nach dem Erreichen des höchsten Fiebers aber beide sich steigern, so dass die Körpertemperatur anf demselben erhöhten Grad verharrt.

Die innige Beziehung des Würmestichfiebers zum Kohlehydrat, besonders zum Leberglykogen baben wir schon oben erwähnt A rons ohn u. Sa ch s ${ }^{35}$ und Hirsch u. Mülle $\mathrm{r}^{36)}$ betonen, dass beim Wärmestichfieber die Temperatur der Leber am höchsten ist.

Was die Veränderungen des Blutzuckergehalts bei Wärmestichfieber betrifft, so beobachtete S e na tor ${ }^{3 i}$ ) beträchtliche Zunahme, aber K un n ${ }^{38}$ ) Eintreten von Hyperglykämie noch vor dem Fiebern, ganz gleich ob das Fieber durch Wärmestich erzeugt wird oder nicht, nach dem Fieber aber lebhafte Verbrennung des Kohlehydrats und Abnahme des Blutzuckerwerts. Morita u. Naito $0^{39}$ äussern sich dahin, dass sich der Blutzuckergehalt bei Wärmestichfieber kaum verändert, und $\mathrm{Jak}$ obowsky, ${ }^{40)}$ dass die Hyperglykämie bei infektiösen Fieberzuständen nicht durch Körpertemperatursteigerung eintritt, sondern durch die Toxinwirkung verursacht werden müsste, weil der Blutzuckergehalt vor dem Wärmestichfieber zunimmt, nach dem Beginn des Fiebers aber allmählich abnimmt und zum normalen Wert zurückkehrt.

Bei unserem Versuche war zu beobachten, dass bei erfolglosem Wärmestich der Blutzuckerwert schon bei der zweiten Blutentnahme beträchtlich vermindert war und danach kaum noch schwankte, während er beim erfolgreichen Wärmestichfieber meist gleich nach dem, Erreichen des höchsten Fiebers beträchtlich zunimmt.

Der Blutzucker vermindert sich scheinbar bei Versuch 1 nach dem Fieber, was aber seiner beträchtlichen Zunahme infolge des Fesselns bei der ersten Blutentnahme zuzuschreiben ist, und beträgt den hohen Wert von $141 \mathrm{mg} / \mathrm{dl}$ auch dann, wenn er nach dem Fieber am stärksten vermindert war, woraus zu ersehen ist, dass der obige Wert deutlich höher ist als der Blutzuckergehalt des Kaninchens bei den oben erwähnten Autoren.

Bei ungefähr $40^{\circ} \mathrm{C}$ Fieber nimmt der Blutzucker, der sich, mit Ausnahme von Versuch 7, meist im Anfangsstadium des höchsten Fiebers vermehrt, schnell ab, während bei demjenigen Versuchsfall (Ver-

33) O d a ira, Tohoka Journ. Exp. Med., 1925, 6, 534.

34) Schultze, Arch. f. exp. Pathol. n. Pharmakol., 1900, 43. 193.

35) Aronsohn u. Sachs, Dtsch. med. Wochenschr, 1884, 823 ,

36) Hirsch a. Mülle r, Dtsch. Arch. f. kl. Med., 1903, 75, 287.

37) Senator, Zeitschr. f. kl. Med., 1909, 67, 253.

38) K u no, Tokyo Irrakkai Zasshi, 1914, 28, 1403.

39) Morita u. N a ito, Tohoku Journ. Exp. Med., 1922, 2, 562.

40) J a k o b o wskg, Upsala läkareförenings forhandl., 1923, 28, 215; ref. in Berichte über d. ges. Physiol.. 1923, 19, 61. 
such 5), dessen Fieber etwa $41^{\circ} \mathrm{C}$ erreichte, die Vermehrung des Blutzuckers noch während 3 Stunden nach dem Erreichen des höchsten Fiebers anhält, und zwar bei einem Fall (Versuch 4), dessen Fieber bis auf ungefähr $42^{\circ} \mathrm{C}$ gelangte, stieg der Blutzuckerspiegel während der weiteren 4 Stunden, soweit wir beobachteten, allmählich immer mehr und zeigte keine Neigung zur Abnahme.

It ${ }^{41)}$ aus unserer Klinik beobachtete beim Kaninchen, dass die Zunahme des Blutzuckers bei Einführung verschiedener Säuren parallel zur Steigerung der $\mathrm{H}^{*}$-Konzentration des Blutes verläuft und bei deren beträchtiicher Steigerung die Erholung des Blutzuckerspiegels langsamer vor sich geht.

Es lässt sich bei unserem Versuch denken, dass die Steigerung der $\mathrm{H}^{*}$-Konzentration des Blutes während der Fieberdauer um so grösser werden muss, je höher das Fieber ist; die Zunahme des Blutzuckergehalts dabei müsste also auch zur Zunahme der $\mathrm{H}^{*}$-Konzentration Beziehung haben, woraufhin dann der Verlauf des Blutzuckerspiegels, wie oben erwähnt, verschieden verlaufen dürften. Daraus ist zu ersehen, dass die Hyperglykämie bei Wärmestichfieber nicht einfach durch die mechanische Reizug des Corpus striatum im Zwischenhirn verursacht wird, sondern auf die sekundäre Veränderung durch Fieber selbst zurückzuführen ist. Ferner weist die Angabe Euler $\cdot{ }^{42}{ }^{42}$ dass das Blutserum bei Stichfieber an Adrenalin reich ist, darauf hin, dass dabei auch Adrenalinhyperglykämie eintritt.

Kein bestimmtes Vermehrungsverhältnis des Blutzuckers gegenüber je $1^{\circ} \mathrm{C}$ Steigerung der Körpertemperatur war zu erkennen, weil der Blutzuckerspiegel schon vor der Ausführung des Wärmestichs ganz verschieden war, je nach dem, ob die Fesselungshyperglykämie früher oder später eintrat.

Die Blutmilchsäure vermehrte sich gleich nach dem Erreichen des höchsten Fiebers am meissten, verminderte sich aber danach allmählich wieder und erreichte in 2-3 Stunden wieder den Wert vor Fieber, was mit den Veränderungen der Milchsäure beim Naphthylaminfieber ganz übereinstimmt.

$\mathrm{K}$ ond $\mathrm{o}^{43}$ hat bomerkt, dass Milchsäurebildung im Organgewebe durch Alkalizusatz gefördet und durch Säurezusatz gehemmt wird. A ki y a ${ }^{44}$ bei Wärmestichfieber, dass im Stadium des steigenden Fiebers Alkalosis, im Stadium des dauernden Fiebers aber Azidosis eintritt. Aus diesen Tatsachen ist zu ersehen, dass im Stadium des steigenden Wärmestichfiebers durch Glykogenolyse, Zucker und Milchsäure gebildet werden, im Stadium des dauernden Fiebers aber die Milchsäurebildung allmählich gehemmt wird bis zu ihrer Abnahme. Iwa-

41) It o, Tohoku Journ. Exp. Med., 1926-27, 8, 75.

42) Euler, Pflìger's Arch., 1927, 217, 699.

43) Kond o, Bioch. Zetschr., 1912, 45, 63.

44) A ki y a, Zeitschr, f. kl. Med, 1928, 108, 312. 
sa $w a^{45}$ beobachtete bedeutende Hemmung der anaeroben Glykolyse nach Wärmestichfieber, und kürzlich haben $\mathrm{K}$ od e ra u. Ad a c hi, ${ }^{46)}$ beim gesunden Hunde beobachtet, dass bei Dauerinfusion von Salzsäure der Zuckerverbrauch und die Milchsäurebildung des Muskels gehemmt werden.

Die Zunahme der Blutmilchsäure für je $1^{\circ} \mathrm{C}$ Körpertemperatursteigerung gleich nach dem Erreichen des höchsten Fiebers beträgt 1,48-18,37 mg/dl; diese grosse Ver'schiedenheit ist ebenso wie beim Naphthylaminfieber.

Da die Abnahme des Leberglykogens bei Wärmestichfieber, wie Rolly $\mathrm{y}^{27)}$ beobachtete, grösser als die im Muskel ist, so ist es zu ersehen, dass der dabei vermehrte Zucker- und Milchsäuregehalt des Bluts grösstenteils, wie einer von uns (A dachi) früher erklärte, von der Spaltung des Leberglykogens herrühren. Andererseits müssen der dabei gebildete Blutzucker und die Blutmilchsäure im Muskel durch genügende $\mathrm{O}_{2}$-Lieferung verbrannt werden. An der Zunahme des $\mathrm{O}_{2}$-Verbrauchs, die Aronsohn u. Sachs, ${ }^{32)}$ und Odaira ${ }^{33)}$ dabei beobachtet haben, muss sich auch diese Verbrennung im Muskel beteiligen.

Beim Vergleich der Kurve des Blutzuckers mit der der Blutmilchsäure bei Wärmestichfieber erkennt man, dass die Blutmilchsäure immer gleich nach dem Erreichen des höchsten Fiebers am höchsten steigt und 2-3 Stunden danach, trotzdem sich das Fieber noch auf derselben Höhe hält, auf den Wert vor Fieber zurückkehrt, während

Fig. 6. (Versuch 4, Wärmestichfieber.)

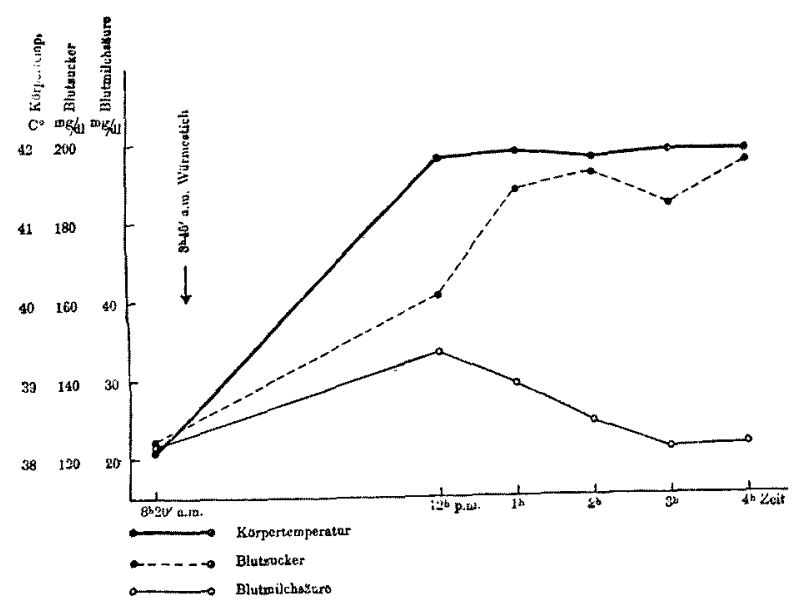

45) I was a wa, Nippon Naikagakkai Zasshi, 1928, 16, 444.

46) Kodera $\mathrm{u}$. A d a chi, wird bald in dieser Zeitschrift publiziert. 
sich der Blutzuckergehalt bei leichtem Fieber gleich nach dem Erreichen des höchsten Fiebers vermehrt und dann allmählich vermin. dert, so dass beide Kurven parallel zueinander verlaufen. Aber beim höheren Fiebern nimmt der Blutzucker auch bei Fieberdauer immer weiter zu, so dass beide Kurven auseinandergehen (Fig. 6 resp. Versuch 4.). Dieser verschiedene Verlauf dürfte daher rühren, dass die Bildung der Milchsäure durch Glykolyse bei leichter Steigerung der $\mathrm{H}^{*}$-Konzentration gehemmt wird, die Zunahme des Blutzuckers infolge Glykogenolyse aber bei leichter Zunahme der $\mathbf{H}^{*}$-Konzentration vorübergehend ist und erst bei ihrer starken Erhöhung der Blutzucker verspätet abnimmt.

\section{Schluss.}

Die Untersuchung über den Einfluss der Fesselung und des Fiebers auf den intermediären Kohlehydratstoffwechsel am gesunden Kaninchen hat nachstehende Ergebnisse gebracht:

1. Durch dauernde Fesselung sinkt die Körpertemperatur und steigt danach nur sehr langsam wieder. Der Blutzucker vermehrt sich allnählich, 3-5 Stunden nach dem Fesseln am meisten und nimmt dann ganz allmählich wieder ab, aber selbst 7 Stunden nach dem Fesseln besteht noch leichte Hyperglykämie. Die Blutmilchsäure nimmt gleich nach dem Fesseln beträchtlich zu, vermindert sich dann allmählich und kehrt 2 Stunden nach dem Fesseln auf den Wert davor zurück, schwankt aber danach nur wenig.

2. Bei Naphthylaminfieber nimint der Blutzucker bald zu, bald $a b$, verändert sich also auf unbestimmte Weise; das dürfte darauf beruhen, dass das Auftreten einer starken Fesselungshyperglykämie die Veränderung des Blutzuckers durch Fieber verdeckt.

3. Bei Naphthylaminfieber vermehrt sich die Blutmilchsäure gleich nach dem Erreichen der grössten Temperatursteiggerung, vermindert sich damn aber allmählich und erreicht 1-3 Stunden nach dem höchsten Fieber wieder den Wert vor Fieber.

4. Bei Wärmestichfieber vermehrt sich der Blutzucker im Anfangsstadium des höchsten Fiebers, vermindert sich dann aber allmählich und zeigt Neigung, auf den Wert vor Fieber zurückzukehren; aber bei hochgradigem Fieber erreicht der vermehrte Blutzucker manchmal nur schwer wieder den Anfangswert.

5. Bei Wärmestichfieber vermehrt sich die Blutmilchsäure gleich nach dem Erreichen des höchsten Fiebers und kommt 2-3 Stunden danach auf den Wert vor Fieber zurück. 